\title{
Pulmonary anatomy in the Nile crocodile and the evolution of unidirectional airflow in Archosauria
}

The lungs of birds have long been known to move air in only one direction during both inspiration and expiration through most of the tubular gas-exchanging bronchi (parabronchi). Recently a similar pattern of airflow has been observed in American alligators, a sister taxon to birds. The pattern of flow appears to be due to the arrangement of the primary and secondary bronchi, which, via their branching angles, generate inspiratory and expiratory aerodynamic valves. Both the anatomical similarity of the avian and alligator lung and the similarity in the patterns of airflow raise the possibility that these features are plesiomorphic for Archosauria and therefore did not evolve in response to selection for flapping flight or an endothermic metabolism, as has been generally assumed. To further test the hypothesis that unidirectional airflow is ancestral for Archosauria, we measured airflow in the lungs of the Nile crocodile (Crocodylus niloticus). As in birds and alligators, air flows cranially to caudally in the cervicoventrobronchus, and caudally to cranially in the dorsobronchi in the lungs of Nile crocodiles. We also visualized the gross anatomy of the primary, secondary and tertiary pulmonary bronchi of C. niloticus using computed tomography (CT) and microCT. The cervicoventrobronchi, cranial dorsobronchi and cranial medial bronchi display similar characteristics to their proposed homologues in the alligator, while there is considerable variation in the tertiary and caudal group bronchi. Our data indicate that the aspects of the crocodilian bronchial tree that maintain the aerodynamic valves and thus generate unidirectional airflow, are ancestral for Archosauria. 


\section{Author Affiliation:}

6

7 Emma R. Schachner, Department of Biology, University of Utah, Salt Lake City, UT, 84112,

8 USA, email: eschachner@gmail.com

9

10 John R. Hutchinson, Structure \& Motion Laboratory, Department of Comparative Biomedical

11 Sciences, The Royal Veterinary College, Hatfield, Hertfordshire, United Kingdom, email:

12 jhutchinson@rvc.ac.uk

13

14 C. G. Farmer, Department of Biology, University of Utah, Salt Lake City, UT, 84112, USA, email:

15 cg.frmr@gmail.com

20 Corresponding Author:

21 Emma R. Schachner, Department of Biology, University of Utah, Salt Lake City, UT, 84112,

22 USA, email: eschachner@gmail.com, phone: 610-724-7583 
23 The evolution of the clade Archosauria is a popular subject of scientific study because of the

24 dramatic evolutionary radiations that characterize it. Archosauria includes many extinct lineages,

25 such as crocodyliforms and other pseudosuchians (ornithosuchids, aetosaurs, and poposaurs),

26 pterosaurs, and non-avian dinosaurs. The latter grade came to dominate numerous niches on

27 land during the Mesozoic Era, but were supplanted by mammals after the

28 Cretaceous-Paleogene extinctions. Whereas birds survived the Mesozoic to become extremely

29 widespread, multiple lineages of crocodyliforms persisted at much lower levels of diversity yet a

30 nonetheless impressive global distribution. Explanations for the archosaur radiation are

31 complicated by a puzzling pattern of faunal turnover. Did archosaurs evolve particular features

32 that gave them a competitive advantage that enabled them to supplant the synapsids, or did

33 they flourish opportunistically in the wake of the massive Permo-Triassic extinctions? Many

34 aspects of the archosaurian radiation, such as morphological variety, rates of morphological

35 character evolution, faunal abundance, and taxonomic diversity, have been examined in an

36 increasingly rigorous manner, providing insight into the patterns and processes underpinning

37 this radiation (Nesbitt 2011). However, these patterns are consistent both with changes that are

38 predicted when a lineage is presented with new ecospace and when it evolves an innovative

39 trait (Brusatte et al. 2010).

40 The respiratory system is the interface between the environment and the internal milieu and is

41 the first step in the oxygen cascade. It is thought to be particularly derived in birds because it

42 consists of air sacs that function as bellows and lungs composed of a series of open-ended

43 tubes (dorso, ventro, and parabronchi) (Duncker 1971). Furthermore, airflow through most of

44 these tubes occurs in one direction during both phases of the respiratory cycle due to the

45 presence of aerodynamic valves (Butler et al. 1988; Wang et al. 1988). Conventional wisdom

46 has attributed the evolution of these features of the avian respiratory system to the high

47 energetic demands of flight (Maina 2000). Alternatively, it is possible that endothermy, rather 
than flight per se, underpins the evolution of these features. Various aspects of the avian respiratory system have been proposed to have evolved in the ornithodiran lineage before the evolution of birds (Huxley 1882; Perry 1992; Bonde and Christiansen 2003; O'Connor and Claessens 2005; Wedel 2007; Sereno et al. 2008; Claessens et al. 2009; O'Connor 2009; Wedel 2009; Butler et al. 2012; Yates et al. 2012). For example, in discussing the dorsal expansion of the lungs in extant archosaurs in 1882, Huxley proposed that, "It seems not improbable that the great height of the bodies and arches of the anterior thoracic vertebrae in some Dinosaurians may be connected with a similar modification of the lungs." The recent discovery of unidirectional airflow in the lungs of alligators (Farmer 2010; Farmer and Sanders $\underline{2010})$ suggests the character of unidirectional airflow through open-ended, tubular gas-exchanging structures is older than the ornithodiran lineage, and predates the evolution of avian style air sacs, having evolved in the common ancestor of the pseudosuchian and ornithodiran lineages. Unidirectional airflow and the structures requisite for aerodynamic valves have been proposed to have arisen in the ectothermic ancestors of these lineages and to have functioned as a means to couple the motion of the beating heart with airflow during periods of breath-holding (apnea) (Farmer 2010). In this scenario, the unidirectional airflow found in birds, which appears to facilitate their ability to fly in hypoxic conditions (Meyer et al. 1981), is an exaptation, having originally served their distant ancestors in a completely different role, that of facilitating gas exchange during apnea.

Levels of atmospheric oxygen have probably played a large role in the evolution of life throughout the Phanerozoic (e.g., Graham et al. 1995; Huey and Ward, 2005; Berner et al. 2007; Kaiser et al. 2007). The archosaur lung may be a key innovation that gave archosaurs a competitive advantage over the synapsids in niches that required highly aerobic metabolisms during the atmospheric hypoxia of the Triassic (Farmer 2010; Farmer and Sanders 2010). The avian lung has long been thought to be an adaptation for the high aerobic demands of flapping 
73 flight (Maina 2000). Furthermore, features of the avian lung, such as unidirectional airflow,

74 appear to improve the efficacy of gas exchange under conditions of hypoxia (Meyer et al. 1981).

75 If pulmonary aerodynamic valves and unidirectional airflow were present in basal archosaurs,

76 these characters could have given the entire lineage a selective advantage under conditions of

77 hypoxia. Thus key innovations in the respiratory system may have enabled the archosaurs to

78 usurp the synapsid dominance in ecomorphological niches that required high aerobic capacities

79 (Farmer 2010).

A considerable amount of work has been done on the macro- and microscopic anatomy

81 of reptilian lungs (Broman 1939; Duncker 1978; Perry and Duncker 1978; Klemm et al. 1979;

82 Perry 1998), including that of crocodilians (Lereboullet 1838; Cuvier 1840; Milani 1897; Perry

83 1988; Perry 1990; Perry 1998). However, less attention has been focused on the anatomy of the

84 crocodilian bronchial tree (Sanders and Farmer 2012) despite long known similarities to avian

85 primary and secondary bronchi (Huxley 1882; Moser 1902; Broman 1939; Boelert 1942; Perry

86 1988; Perry 1989; Perry 1992; Perry 2001; Farmer 2010; Farmer and Sanders 2010; Sanders

87 and Farmer 2012). For example, in describing the crocodile lung Huxley (1882) states, "Each

88 bronchus is continued directly backwards into a wide canal, which dilates into an oval sac-like

89 cavity at the posterior end of the lung, representing the mesobronchium with the posterior

90 air-sac in birds. In the dorsal and mesial wall of the mesobronchium there are five or six

91 apertures, which lead into as many canals, representing the entobronchia (ventrobronchi) in

92 birds. These pass, the anterior two almost directly forwards, and the others more or less

93 obliquely, to the dorsal margin; and they lie quite superficially on the mesial face of the lung.

94 The first is very much larger than the others, and ends in a dilatation at the anterior end of the

95 lung. It is united with the second by transverse branches. Along the ventral margin of the lung

96 there are four sac-like chambers, which communicate, in the case of the two anterior, with the

97 entobronchia, and, in the case of the two posterior, with the mesobronchium. Finally, there are 
98 two very large canals, external to these, which communicate with the mesobronchium by large

99 apertures in its dorsal wall, and give off branches to the outer face of the lung, representing the

100 ectobronchial (dorsobronchial) system of birds. The orifices with which the surfaces of all these

101 canals, except the anterior half of the mesobronchium, are thickly set, lead into depressions,

102 which are often so deep as to become cylindrical passages, simulating the parabronchia of

103 birds. Thus, notwithstanding all the points of difference, there is a fundamental resemblance

104 between the respiratory organs of Birds and those of Crocodiles, pointing to some common form

105 (doubtles exemplified by some of the extinct Dinosauria), of which both are modifications." To

106 date three studies have measured airflow patterns in the American alligator (Alligator

107 mississippiensis, clade Alligatoridae) (Bickler et al. 1985; Farmer 2010; Farmer and Sanders

108 2010). Using scintigraphy, Bickler and colleagues (1985) described a radial spread of gas from

109 the intrapulmonary bronchus into a multicameral alligator lung, and tidal airflow. In contrast,

110 direct measurements of airflow in A. mississippiensis demonstrated that gases move

111 unidirectionally through most of the secondary bronchi (Farmer and Sanders 2010). These data

112 indicated that the previous understanding of the relationship between anatomical architecture

113 and airflow patterns in the lung of Alligator was incorrect. The lung is not composed of multiple

114 chambers (multicameral) that end blindly, but of open ended tubes. Furthermore, the presence

115 of unidirectional airflow in crocodilians suggests that this pattern of airflow is basal for the entire

116 clade Archosauria.

117 To gain insight into basal archosaur pulmonary anatomy, and to elucidate how and why the

118 lungs of birds and those of the American alligator diverged, requires the careful study of a range

119 of crocodilian and avian species. Whereas numerous studies are available for both anatomical

120 and physiological aspects of avian lungs (Duncker 1971; Brackenbury 1972; Maina and

121 Nathaniel 2001; Maina 2006; Farmer and Sanders 2010), there are few studies of the

122 crocodilian respiratory system, particularly studies that combine physiological and anatomical 
123 measurements. The clade Crocodylia is composed of at least two major lineages: Alligatoroidea,

124 which includes the two extant alligator species and seven extant caiman species and

125 Crocodyloidea, which includes the 13+ extant species of crocodiles. However, the phylogenetic

126 position of a potential third lineage, the Gavialidae (gharials), remains controversial (lying

127 outside Alligatoroidea + Crocodyloidea, or within the latter clade (Brochu 1997; Gatesy et al.

128 2003; Oaks 2011). Identification of key features that are common to all the crocodilian lineages

129 and those that vary interspecifically necessitates detailed study of species from each lineage.

130 Here, we report the results of detailed study on the anatomy and airflow patterns in the lungs of

131 the Nile crocodile (Crocodylus niloticus), the first such analysis of a non-alligatoroid crocodylian.

132

\section{MATERIALS AND METHODS}

We collected data from seven specimens of Crocodylus niloticus and five specimens of

Alligator mississippiensis for comparison. Approval for this study was granted from the

University of Utah Institutional Animal Care and Use Committee (IACUC), protocol number

10-12003. C. niloticus were obtained post mortem (varied, natural causes but no respiratory pathology) from the conservation and breeding center "La ferme aux crocodiles" (Pierrelatte, France), with specimen identifiers FNC6 (10.1 kg), NNC1 (3.2 kg), NNC3 (1.01 kg), NNC4 (14.6 $\mathrm{kg})$, NNC5 $(0.5 \mathrm{~kg})$, NNC6 $(0.8 \mathrm{~kg})$, and NNC9 $(0.58 \mathrm{~kg})$. The five alligators were obtained from

141 the Rockefeller Wildlife Refuge in Louisiana: $2.3 \mathrm{~kg}, 3.6 \mathrm{~kg}, 5.4 \mathrm{~kg}, 3.6 \mathrm{~kg}, 11 \mathrm{~kg}$, and 64 inches

142 long (mass unknown). The C. niloticus lungs were excised and soaked in an iodine potassium

143 iodide (I2KI) solution at concentrations varying from $2.25-3.75 \%$ (Jeffery et al. 2011) prior to the

144 CT scans. NNC5 and NNC6 were inflated and scanned in a medical grade CT unit at the Royal

145 Veterinary College, London at 90kVp and 133MA with a slice thickness of $0.75 \mathrm{~mm}$; NNC9 was

146 scanned in a micro CT unit at the University of Cambridge with a slice width of $0.0816 \mathrm{~mm}$. We

147 imaged live unsedated adult alligators during a natural apnea phase at the University of Utah

148 Medical Center using a 164 slice dual energy Siemens SOMATOM Definition computed 
149 tomography unit. Image acquisition parameters: slice thickness $=0.6 \mathrm{~mm}$ and $1 \mathrm{~mm}, \mathrm{kVp} 120$,

150 MA 200. The pulmonary bronchi were segmented into a 3D model from DICOM image files with

151 the visualization software Avizo 7.

152 To measure flow, dual heated thermistor airflow probes were individually implanted in

153 each of the secondary bronchi (Fig. 1) after the anatomy was mapped out using computed

154 tomography and Avizo 7.0 software. The probe was connected to an air flow meter (HEC 132C

155 Thermistor Flowmeter, Hector Engineering Co., Inc., Elletsville, IN), and the signal transformed

156 from analog to digital (Biopac Systems Inc, Goleta, CA), and then recorded on a Maclntosh G4

157 Powerbook laptop using AcqKnowledge software (Biopac Systems Inc, Goleta, CA). Airflow in

158 and out of the trachea was measured with a pneumotach (Hans Rudolph Inc. Shawnee, KS).

159 Measuring airflow in excised lungs in crocodilians has been validated and produces the same

160 results as in vivo experiments (Farmer 2010; Farmer and Sanders 2010); thus only ex vivo

161 lungs were used here. Once the probe was in place (Fig. 1), air was pushed into the excised

162 lung using a syringe to measure airflow in and out of the primary and secondary bronchi.

164 RESULTS

165

166 Trachea and carina The trachea contains cartilaginous rings, and is very distensible along its

167 long axis (Fig. 2A). It is centered approximately ventral to the esophagus in smaller individuals

$168(\leq 0.5 \mathrm{~kg})$ but lies to one side in the larger animals $(5-15 \mathrm{~kg})$. In the smaller specimens, the

169 carina is located just cranial to the outlet of the great vessels from the pericardium. In the larger

170 animals, it is positioned much more proximally and due to an elongation of the primary bronchi,

171 the trachea forms a distinct loop (Fig. 2B).

172

173 Primary bronchi The primary bronchi are composed of three distinct parts: the extrapulmonary

174 primary bronchi, the cartilaginous intrapulmonary primary bronchi and the non-cartilaginous 
175 intrapulmonary primary bronchi. The extrapulmonary primary bronchus enters the lung

176 ventro-medially at approximately one third the length of the lung from its apex, and courses in a

177 drawn out S-shaped curve laterally, caudally, and dorsally. In all of the specimens examined,

178 the non-cartilaginous portion of the intrapulmonary primary bronchus broadens significantly to

179 become at least twice as wide as the cartilaginous region as it extends caudally; it then loops

180 medially at the caudal end of the lung generating a distinctive hook-like bronchus. At the caudal

181 margin of the hook in all specimens, the primary bronchi balloon out caudally into sub-equal

182 caudally positioned sac-like structures, in both lungs (Fig. 3). The caudal region of the lung in

183 Crocodylus niloticus is less vascularized than the dorsal regions and as a result is likely less

184 involved in gas exchange (Perry 1990).

185

Secondary bronchi

There are several types of secondary bronchi (Fig. 4). They differ due to the location within the lung and by their airflow patterns.

Cervical ventral bronchi (CVB; D1) The most proximal and first ostium on the primary

191 bronchus is very close to the hilus and opens on a largely lateral location on the primary

192 bronchus into a conical vestibule. This cone makes a hairpin turn into a cranially directed and

193 large diameter bronchus. This bronchus is the ventrobronchus (the CVB), or D1 (the D1 is from

194 Broman's (Broman 1939) identification as the first dorsal branch off of the primary bronchus)

195 (Fig. 5A-D; 5A-D). The CVB arches cranially so that the main body of the bronchus lies almost

196 parallel to the trachea. There is some variability in the overall morphology of the CVB from

197 individual to individual and even between the right to left lungs. In some individuals (e.g., NNC9;

198 Fig. 5A-D; 6A-D), there is a large hook on the distal tip of the CVB that arches dorsally then

199 caudally towards the distal tip of D2. 
201 Dorsobronchi (D2-X) The dorsobronchi arise sequentially via large oval-shaped openings

202 (termed macroostia (Sanders and Farmer 2012)) from the dorsal and dorsolateral surface of the 203 cartilaginous intrapulmonary primary bronchi and variably up to one half of the proximal part of 204 the non-cartilaginous intrapulmonary primary bronchi. Along with the CVB, they are the largest 205 bronchi in the lung, arching dorsally and then cranially (Fig. 5A, B). Crocodylus niloticus has 206 between four and six dorsobronchi; however, there is individual variation, as well as bilateral

207 variation between the right and left sides with regard to both number and specific bronchial 208 morphology. In all specimens, D2-D4 are long tubular bronchi with a wide base that arch 209 dorsally and then run cranially towards the apex of the lung. The more caudal dorsobronchi

210 (D5-7) run dorsally or dorsolaterally from their origin and are generally half the length

211 (longitudinally) of the proceeding three. They also often exhibit more branching, intermediate

212 between D2-4 and the laterobronchi in one specimen (NNC9).

213

214 M bronchi (M1-X) The M, or medial bronchi exhibit a similar morphological pattern to that of the 215 dorsobronchi, but have a medial origin from the cartilaginous intrapulmonary primary bronchi.

216 There is more bilateral asymmetry in M bronchi between the right and left lungs in Crocodylus

217 niloticus, with variation in both the number of branches (six to eight) and overall branch

218 morphology (Fig. 5C, D). In all three specimens, M1 is the second branch off of the primary

219 bronchus. It maintains a long, tubular anatomy and runs dorsocranially in unison with D2. The

220 subsequent branches vary from individual to individual, but follow an overall trend: the more

221 cranial branches are tubular and have a wide, rounded base; the middle bronchi pass dorsally

222 giving off both cranial and caudal forks; the caudal M bronchi arch caudally and then

223 caudoventrally terminating in a sac-like tip.

224

225 Laterobronchi Multiple small ostia along the ventral and lateral surface of the cartilaginous 226 intrapulmonary primary bronchi open up into sac-like secondary bronchi (Fig. 6A-D). These 
227 laterobronchi have very small, constricted openings that balloon out into large dead-end chambers containing multiple finger-like protrusions that extend in all directions. The

229 laterobronchi vary in size, number and morphology between the right and left lungs as well as

230 among individual specimens.

231

232 Caudal Group Bronchi (CGB) The number and morphology of the CGB are very variable

233 across the individual animals examined; however, there were a few relevant invariable

234 characters. The CGB are the most numerous type of secondary bronchus, maintain a tube-like

235 morphology, and branch in all directions from the non-cartilaginous primary bronchi (Fig. 6A-D).

236 In Crocodylus niloticus, the CGB extend caudally from the hook of the primary bronchus to the

237 caudal margin of the lung. Like the alligator, these bronchi are significantly less vascularized

238 than the dorsobronchi and cranial M bronchi.

241 Large diameter tertiary bronchi The CVB and D2-4 all give off major tertiary branches, the majority arising from the base and proximal third of all five secondary bronchi (Fig. 4B). The largest of these tertiary branches run cranioventrally from all four secondary bronchi to the ventral surface of the lung where they then balloon out much like the laterobronchi. These tertiary bronchi are non-contiguous with the laterobronchi but generate a sequence of chamber-like air sacs that occupy the mid-to caudoventral region of the lungs. Smaller more tubular tertiary bronchi emerge from all of the dorsobronchi and $\mathrm{M}$ bronchi along their entire length. In both lungs, tertiary bronchi branch off of the $\mathrm{M}$ bronchi to contribute to the cardiac lobes (=pericardiac air sacs). There are (variably) three to four bronchi that contribute to the left and right cardiac lobes in Crocodylus niloticus, which adhere to the dorsal surface of the 251 pericardium. 
253 Small diameter anastomosing bronchi (parabronchi)- The parabronchi are small tubular

254 bronchi that interconnect the secondary bronchi forming a loop between the dorsobronchi and

255 the CVB (Fig. 7B, C). These small parabronchi also variably anastamose with adjacent large

256 secondary bronchi. There appears to be a diastema between the origination of the CVB and first

257 dorsobronchus (D2) and the emergence of the first parabronchus interconnecting the two

258 bronchi.

259

260

Airflow patterns in the major secondary bronchi Airflow was measured in four of the large

261 secondary bronchi in five individual specimens of Crocodylus niloticus. In the three dorsobronchi

262 that arise sequentially along the primary bronchi caudal to the CVB (D2-4), air travels caudally

263 to cranially during both phases of the respiratory cycle (Fig. 8A-E; 9). In the CVB, the first

264 bronchus to arise off of the primary bronchus, air flowed cranially to caudally during both phases

265 of respiration in all specimens (Fig. 8G, H; 9) (inhalation and exhalation). The dorsobronchi

266 connect to the CVB via the parabronchi (Fig. 7), generating the continuous loop that maintains

267 this airflow pattern (Fig. 10).

268

269

270

DISCUSSION

271

272 Gross anatomy - A broad range of terminology has been used for the different pulmonary

273 structures in the lungs of reptiles (Broman 1939; Perry 1998; Sanders and Farmer 2012). The

274 trachea and extrapulmonary primary bronchi are nearly universal terms, but considerable

275 variation exists in the terminology used to describe the intrapulmonary bronchus

276 (mesobronchium by Huxley (1882)) and the second and third generations of branching of the

277 avian lung. Based upon hypotheses of homology between alligators and birds proposed by

278 Sanders and Farmer (2012), observed morphological and functional similarities between 
Crocodylus niloticus, the American alligator and birds, we have chosen to adopt their nomenclature with a few adjustments that incorporate the original developmental terms that 281 Broman (1939) gave to the secondary bronchi in the alligator. Huxley (1882) and others have 282 tended to name the secondary bronchi of birds according to the topological regions they come 283 to occupy. Thus Huxley described entobronchia that come to occupy the ventral and medial 284 portions of the lung. These were termed "bronches diaphragmatique" of (Sappey 1847) because 285 of their association with the avian diaphragm and are referred to as "ventrobronchi" by Duncker 286 (1971) because they occupy the ventral lung regions. The ventrobronchi have their origin in 287 openings in the proximal and dorsal part of the intrapulmonary bronchus. The first

288 ventrobronchus curves sharply round the entrance of the intrapulmonary bronchus and courses 289 cranially to occupy the cranioventral portion of the lung and to communicate with the cranial set 290 of air sacs. The other ventrobronchi also come to occupy caudal and mesial regions of the lung. In contrast the "ectobronchi" of Huxely, "bronches costales" of Sappey and "dorsobronchi" of

292 Duncker are six or seven in number and run laterally and dorsally toward the lateral or costal 293 face of the lung. A third group of bronchi that come to occupy the caudolateral portions of the 294 lung were termed laterobronchi by later authors (e.g. (Duncker 1971; Duncker 1972). Their ostia 295 arise at the same level of the intrapulmonary bronchus as the dorsobronchial ostia. Arising from 296 walls of the ecto- and entobronchi are round apertures that lead into canals that course more or 297 less at right angles to the surfaces of the bronchi. These canals sometimes anastomose with 298 each other and were termed parabronchi ("canaux tertiaries", Cuvier 1840). A further set of 299 tubes arising from the parabronchi were termed intercapillary air-passages (air capillaries, 300 Duncker 1971).

301 Much of the gross anatomy of our specimens is consistent with previous work on Nile 302 crocodiles (Perry 1988; Mushonga and Horowitz 1996) but with several significant exceptions.

303 As previously reported, the lungs, heart and great vessels, and esophagus occupy the cranial

304 half of the body cavity. Dorsally and cranioventrally, the lung is rounded (Fig. 1B; 2A; 3A); 
305

306

307

308

309

310

311

312

313

314

315

316

317

318

319

320

321

322

323

324

325

326

327 Branching patterns - Descriptions of the conducting airways of birds and mammals have

328 relied on terminology that relates in part to the degree of branching that has taken place.

329 However, to fully understand the branching pattern requires detailed knowledge of the 
330 development of the airways (Metzger et al. 2008), which is lacking for crocodilians, and so this

331 terminology can be misleading.

332

333 Comparison with Alligator mississippiensis - The overall similarity between the primary,

334 secondary, and tertiary bronchi of Crocodylus niloticus and A. mississippiensis is striking (Fig.

$33511 ; 12)$, suggesting similar genetic control underpinning the branching patterns of the major

336 bronchi in Crocodylia. The anatomy and position of the CVB (D1) and D2-4 are distinctly similar

337 in all specimens of $C$. niloticus and that of $A$. mississippiensis (Sanders and Farmer 2012) (Fig.

338 11; 12). The proximal $\mathrm{M}$ branches (bronchi) are also similar in both taxa. This may be due to the

339 importance of these bronchi in maintaining the integrity of the aerodynamic valve. Another

340 distinct similarity between A. mississippiensis and C. niloticus is the hook the terminal end of

341 the primary bronchus and the caudally extending saccular structure (see Fig. 3).

342 The major differences between the two taxa are subtle, yet suggestive of which

343 pulmonary characters within Crocodylia may be plastic and which are conserved and thus

344 putatively ancestral for the group. Crocodylus niloticus consistently has both more D and M

345 branches than the alligator, as well as significantly more caudal group bronchi (CGB). The CGB

346 are also evenly distributed around the non-cartilaginous intrapulmonary primary bronchus in $C$.

347 niloticus, whereas they are primarily restricted to the ventrolateral surface in Alligator

348 mississippiensis. Farmer and Sanders (2010) identified some large bronchi arising from the

349 dorsal surface of the primary bronchus in the alligator as CGB. However, we consider that these

350 are actually caudal dorsobronchi due to their large ostia, overall morphology, and dorsocranial

351 orientation. Aside from the number of bronchi, the most visible difference between the two taxa

352 is the topography of the tertiary bronchi. In C. niloticus the major tertiary branches of the CVB

353 and D2-4 form an anatomical topology similar to that of the avian laterobronchi; (i.e., they run

354 ventrally and branch into a multichambered sac-like structure). In A. mississippiensis, the major

355 tertiary branches of the first four secondary bronchi are tube-like and run cranially in unison with 
356 their parent branches. Some alligators also have accessory branches emanating from the CVB

357 that have not been observed in C. niloticus.

358 Our observations lead us to infer that certain aspects of the crocodilian bronchial tree

359 are more plastic than others, particularly the number of secondary bronchi and the morphology

360 of the tertiary bronchi. The origin and anatomy of the ventrobronchus (the CVB) and the

361 dorsobronchi appear to be key features in the aerodynamic valves in both the Nile crocodile and

362 the American alligator, whereas the tertiary bronchi and the CGB are more variable in form. Due

363 to the functional relationship between the CVB and the dorsobronchi, we predict that this

364 morphology will be present in other crocodilians, although Gavialoidea remains an important,

365 although difficult to access, target of study.

366

367 Proposed homologies with the avian lung - A discussion of hypothesized homologies between the embryonic and juvenile lungs of the American alligator and the chicken were presented in great detail by Sanders and Farmer (2012) and so an extensive review will not be given here. However, a few anatomical characters that may be important to maintaining unidirectional flow will be discussed here as well as certain aspects of the lung relevant to the evolution of the archosaurian respiratory system. A diagrammatic comparison of the proposed homologies in the crocodilian lung and the avian lung are presented in Fig. 13.

The cervical ventral bronchus (CVB) - Sanders and Farmer (2012) proposed that the

CVB of the alligator was homologous to the embryonic avian cervical air sac, the lateral moiety of the interclavicular sac, and the first ventrobronchus, based upon developmental data in crocodilians (Broman 1939), the chicken (Locy and Larsell 1916), and anatomical data in the juvenile alligator, as well as direct measurements of flow in the CVB that correspond to flow patterns in adult birds in these regions of the lung (Brackenbury 1971; Brackenbury 1972;

380 Brackenbury 1987). These data are supported by both the anatomy and the direction of airflow 381 observed in the CVB of Crocodylus niloticus (Fig. 8; 10; 13). 
niloticus are anatomically similar to those of the bird and airflow patterns are similar in that they

384 flow caudally to cranially in both taxa (Fig. 13; Duncker 1971; Farmer and Sanders 2010).

385 The parabronchi - The anastomosing tubular structures connecting the dorsobronchi

386 with the CVB in Crocodylus niloticus were originally identified as possible homologues of the

387 avian parabronchi by Huxley (1882). The structures found in C. niloticus are in both the same

388 anatomical position as the parabronchi in birds and serving the same function (connecting the

389 dorsobronchi with the ventrobronchi and facilitating flow between the former and the latter) (Fig.

$3907 ; 10 ; 13)$. Parabronchial diameter in birds varies according to species, ranging from $0.05 \mathrm{~mm}$ in

391 diameter in hummingbirds to $2 \mathrm{~mm}$ in coot, chicken, king penguin, and mute swan (Duncker

392 1971). The structures identified in the $0.5 \mathrm{~kg}$ and $0.58 \mathrm{~kg}$ specimens of $C$. niloticus examined for

393 this study were approximately $1.8 \mathrm{~mm}$ in diameter, within the size range found in birds.

The caudoventral saccular region - The primary bronchus continues caudally,

395

396

397

398

399

400

401

402

403

404

405

406

407

widening into a canal that gives rise on its ventral and lateral surface to numerous small ostia that open into sac-like structures making up the ventral and lateral portion of the lung. Further caudally, the primary bronchus balloons into a variable number of sac-like chambers that make up the most caudal aspect of the lung (Fig. 3). Both the ventral and lateral structures and the caudal structures are less vascularized and less morphologically suited to gas exchange than the dorsal regions (Perry 1990) occupied by the dorsobronchi (Fig. 13). As proposed for Alligator by Sanders and Farmer (2012), these bronchi are likely homologous to the avian laterobronchi and the caudal thoracic and abdominal air sacs (Fig. 13). This morphology is also present in Alligator mississippiensis (Sanders and Farmer 2012).

In chickens, the abdominal air sacs are expansions of the distal tip of the primary bronchus, whereas the other air sacs all arise from secondary bronchi (Locy and Larsell 1916). In some adult birds, such as chickens, this morphology becomes greatly exaggerated as the abdominal air sac develops into a massive sac. In contrast, in other birds such as the kiwi, the 
408

409

410

411

412

413

414

415

416

417

418

419

420

421

422

423

424

425

426

427

428

429

430

431

432 433

abdominal air sac is so diminutive that it is difficult to find. Indeed Richard Owen proposed it was lacking altogether, but Thomas Huxley identified a vestigial nub (Huxley 1882).

While there is indeed evidence to suggest homology, the saccular regions of the Nile crocodile are anatomically very different from the air sacs of birds. In birds there are one or more narrow ostia that lead from either the secondary bronchi or the primary bronchus (in the case of the abdominal sac) into the sac. The caudal thoracic sacs and the cranial sacs are contained within the cavum subpulmonale, which is bounded dorsally by the horizontal septum (the avian diaphragm) and ventrally in part by the oblique septum (Huxley 1882; Duncker 1971), whereas the abdominal sac is bounded by the oblique septum and occupies the lateral regions caudal to the cavum subpulmonale (Fig. 13). These septa are formed by the invasion of the pulmonary fold by the lateral thoracic air sacs, which split apart the pulmonary fold into two layers so that the two separate septa are formed: the horizontal (sacco-pleural membrane = avian pulmonary diaphragm) and the oblique septum (thoracoabdominal diaphragm or sacco-peritoneal membrane). In the crocodile, the saccular regions are not projections from the rest of the lung so that the entire lung is readily dissected from the surrounding organs and has an outer contour that is smooth and loaf-like (Fig 1B; 2A). Importantly, although lacking in crocodilians, pulmonary diverticula containing little or no gas-exchange parenchyma and that are reminiscent in ways of avian air sacs exist in numerous squamates, including varanids and chameleons (e.g., Milani 1894; Perry 1998). In summary, although we homologize the saccular caudoventral regions of the lungs of the Nile crocodile with the laterobronchi and caudal air sacs of birds and the first dorsal bronchus of the Nile crocodile with the cervical air sac and lateral moiety of the interclavicular sac as well as the first avian ventrobronchus, we emphasize that these saccular regions are very distinct in birds and crocodilians, just as the wing of a bird is distinct from the arm of a human. Both are forelimbs that arise embryologically from the same tissues in the same region of the embryo, but they form into distinct structures as they mature. 
434 Patterns of airflow - As in extant birds (Butler et al. 1988; Wang et al. 1988) and Alligator

435 mississippiensis (Farmer and Sanders 2010; Sanders and Farmer 2012), no mechanical valves

436 or sphincters were found in Crocodylus niloticus. The conversion of the tidal flow that is

437 entering and leaving the trachea to unidirectional airflow within the lungs in Crocodylus niloticus

438 appears to be produced by the geometry of the primary and secondary bronchi (Fig. 9; 10). Our

439 data suggest it is this bronchial arrangement that generates the inspiratory and expiratory

440 aerodynamic valves. This has been verified in both $C$. niloticus and $A$. mississippiensis via gross

441 dissection, $\mu \mathrm{CT}$ and medical grade $\mathrm{CT}$. In the avian lung, unidirectional airflow in the secondary

442 bronchi occurs via an inspiratory valve in which flow patterns are maintained by the branching

443 angles of the primary and secondary bronchi and convective initial forces (Butler et al. 1988). A

444 similar and perhaps homologous mechanism is likely functioning in C. niloticus. The hairpin turn

445 of the CVB off of the primary bronchus, along with the anastomising parabronchi linking the CVB to the dorsobronchi, provides an architectural arrangement similar to that in the bird (Fig.

10) and this geometry is consistent with the mechanism for the aerodynamic valves proposed

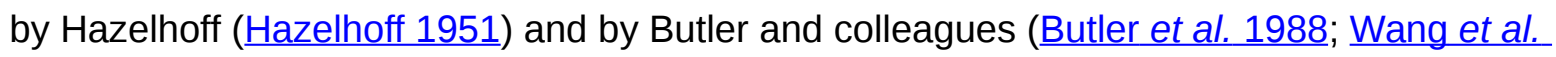
1988). Also, the close proximity of the cardiac bronchi has been hypothesized to be involved with unidirectional cardiogenic airflow in Alligator mississippiensis (Farmer 2010) and due to anatomical similarities a similar mechanism may be occurring in $C$. niloticus. The specific topography of the saccular regions of the lungs probably play little, if any, role in the crocodilian aeorodynamic valve, which is similar to the situation in birds.

In birds, the air sacs serve as ventilatory bellows and storage reservoirs that move air

455 through the primary and secondary bronchi; however, the shape of these sacs do not play any

456 known role in generating the direction of flow within the gas-exchanging portion of the lung.

457 Brackenbury et al. (1989) demonstrated by experimentally occluding the thoracic sacs (= the 458 cranial and caudal thoracic sacs) in adult White Leghorn chickens that these sacs had almost

459 no effect upon the ability of the bird to regulate intrapulmonary airflow during both resting 
460

461

462

463

464

465

466

467

468

469

470

471

472

473

474

475

476

477

478

479

480

481

482

483

484

485

conditions and exercise. In a second set of experiments Brackenbury and Amaku (1990)

occluded both pairs of thoracic sacs and the abdominal air sacs $(\approx 70 \%$ of tidal volume) resulting in a diminished respiratory function but no effect on inspiratory valving, thus demonstrating these air sacs collectively play little role in flow patterns within the gas exchanging lung. That is, aerodynamic valving is not dependent on the presence, location or topography of thoracic (cranial and caudal) and abdominal air sacs.

Origin and evolution of unidirectional airflow - The data of the bronchial topography and patterns of airflow in Nile crocodiles indicate that key features of the respiratory system are shared with both American alligators and birds. The most parsimonious interpretation of these observations is that these features were present in the common ancestor of birds and crocodilians (Archosauria) and were retained in both lineages. These observations are important for several other reasons. They corroborate the hypothesis that the topography of the bronchial passages themselves form the aerodynamic valves that transform the tidal flow that is entering and leaving the trachea into unidirectional flow within the lung (Dotterweich 1936;

Hazelhoff 1951; King 1966; Brackenbury 1971; Duncker 1971; Brackenbury 1972; Brackenbury 1979; Brackenbury 1987; Butler et al. 1988; Wang et al. 1988). Furthermore, they demonstrate that avian style air sacs are not required for flow-through lungs as has been proposed (O'Connor and Claessens 2005) or for unidirectional flow (see below for a discussion of the difference in terminology of "flow-through" lung, as used by O'Connor and Claessens (2005) and in the physiological literature). This raises the interesting question of the selective driver(s) that might have originally favored the evolution of avian air sacs (Farmer 2006). For example, avian air sacs may play important roles in sound production (Plummer and Goller 2008), controlling pitch and roll during slow speed aerial maneuvers (Farmer 2006), or preventing hypocapnia during panting (Brackenbury, 1971). 
486 Terminological turmoil: Comparative physiologists have long used the phrase "unidirectional

487 flow in the lungs" and "flow-through lungs" synonymously. For example, Brown, et al. (1997)

488 state:

489

490

491

492

493

494

495

496

However, the phrases were not used synonymously in the 2005 publication by O'Connor and

Claessens (pers. comm, P. O'Connor, 2013), thus creating confusion because this deviation from standard usage was not stated in the text. The authors stated (O'Connor and Claessens,

2005: pg. 255) "Although our model does not predict the specific type of intrapulmonary air flow in

500 non-avian theropods (unidirectional vs. bidirectional), it does establish both pulmonary and skeletal

501 prerequisites required for flow-through ventilation." Our understanding is that O'Connor and

Claessens did not mean to communicate that they thought unidirectional flow was present

503

inside the lungs of theropod dinosaurs, but rather they meant that these animals had air flowing

504 through the lung from one region differentially to another region, thus creating a new definition

505

for the term "flow-through lungs". By this definition, snakes and other squamates, turtles,

506 amphibians, and lung-breathing fish could be considered to have "flow-through" lungs as they all

507 have regional differences in the distribution of gas exchanging parenchyma within the lung (e.g., 508 Milani 1894; 1897; Perry and Duncker, 1978; Perry, 1989; 1998; Wallach, 1998). However, it 509 would be unusual for respiratory physiologists to refer to these lungs as "flow-through", as this

510 term is normally reserved for the avian lung and means that the air flows unidirectionally through

511 the parabronchi. Here, we exclusively use the standard definition. 
513 Pneumaticity, air sacs, lung efficiency, metabolism, and patterns of air flow in fossil taxa

514 There has been considerable attention given to reconstructing avian-like air sacs in extinct

515 archosaurs based upon patterns of pneumaticity (e.g., Benson et al., 2011; Butler et al., 2012;

516 Claessens et al., 2009; O'Connor and Claessens, 2005; O'Connor, 2006; Sereno et al., 2008;

517 Wedel, 2003; 2007; 2009; Witmer, 1997). It has been suggested that regions of the postcranial

518 axial skeleton are invariably and unambiguously pneumatized by specific air sacs or lungs in

519 birds, and that these patterns of pneumatization can therefore serve as osteological correlates

520 to interpret and reconstruct the presence of specific air sacs in extinct archosaurs (O'Connor

521 and Claessens, 2005; O'Connor, 2006; 2009; Wedel, 2006; 2007; 2009). Furthermore, these

522 patterns of pneumaticity have been purported to be indicative of patterns of airflow throughout

523 the bronchial tree of extinct archosaurs, the 'efficiency' of their lungs, their metabolic capacities,

524 and their thermoregulatory strategies (e.g., Benson et al., 2011; O'Connor and Claessens,

525 2005; Wedel, 2003). The term 'efficiency' normally refers to the energy obtained out of a system

526 per unit of energy put in, yet pneumaticity itself has never been quantitatively linked to

527 measures of actual efficiency, whether considered to be oxygen extraction (Dejours, 1981) or

528 ventilatory equivalent (rate of ventilation/rate of oxygen consumption). A full review of this body

529 of literature is not possible here, but we briefly discuss several recent studies. Based on the

530 presence or absence of postcranial pneumaticity and an array of vertebral laminae and fossae,

531 Butler et al., (2012) proposed that pulmonary air sacs were present in the common ancestor of

532 Ornithodira and were subsequently lost in ornithischian and other members of the clade.

533 Benson et al. (2011) concluded that pneumatization in non-volant maniraptoran nonavian

534 theropods evolved in association with an elevated metabolic rate and "high-performance"

535 endothermy. Wedel $(2007 ; 2009)$ concluded that air sac-driven pulmonary ventilation was

536 ancestral for Saurischia based on the presence of vertebral pneumaticity in various sauropods

537 and theropods. Other osteological features, besides pneumaticity, have been used to try to 
538

539

540

541

542

543

544

545

546

547

548

549

550

551

552

553

554

555

556

557

558

559

560

561

562

563

reconstruct pulmonary anatomy. For example, Schachner et al. (2009; 2011) used the costal and vertebral anatomy of a number of theropods and other dinosauriform archosaurs, as well as the rib anatomy of selected extant taxa, to retrodict the presence of dorsally immobile avian-like lungs. Many other studies have also tried to use pneumaticity to sort out respiratory anatomy and the presence or absence of specific patterns of flow in the lungs of extinct vertebrates (e.g., O'Connor and Claessens, 2006; O'Connor, 2006; 2009; Wedel, 2006; 2007; 2009). Yet postcranial pneumaticity has been purported to be equivocal evidence at best for patterns of air flow, lung efficiency, thermoregulatory strategies, and exercise capacities because pneumaticity has no known function in respiration or gas exchange (Farmer, 2006). Here we have shown that Nile crocodiles neither have postcranial pneumaticity nor air sacs and yet have lungs with truly flow-through ventilation. Hence unidirectional ventilatory flow (a flow-through lung in physiological terms) is possible in an ectothermic animal without pneumaticity and without air sacs. This emphasizes that bronchial anatomy, air sac anatomy, and ventilatory patterns can be decoupled from each other in archosaurs and should not be presumed to be correlated in simple ways.

\section{Acknowledgements}

We thank staff of the Structure \& Motion Laboratory for assistance, as well as radiographic support and advice from Renate Weller and Victoria Watts, La Ferme aux Crocodiles;

particularly Samuel Martin, Eric Fernandez, and Adrien Tomas; for their provision of crocodile cadavers for usage in this study, and Jason Bourke for assistance with Avizo. We thank P.

O'Connor and M. Wedel for two very helpful reviews that greatly improved the manuscript, and we thank editor Andrew Farke for his guidance. We would also like to thank R. M. Elsey of the Rockefeller Wildlife Refuge in Louisiana for providing the American alligators for this study.

E.R.S. is an American Association of Anatomists Scholar and this research was in part funded by the American Association of Anatomists. Additional funding to E.R.S. was provided by the 
564 American Philosophical Society. Funding to C.G.F. was provided by the National Science

565 Foundation (IOS -1055080 and IOS-0818973).

566

567

568 Figure Legends:

569

570 Fig. 1. Excised lungs from a $3.1 \mathrm{~kg}$ Nile crocodile (NNC1; Crocodylus niloticus) demonstrating

571 probe placement, head is to the right. A) Lungs in ventral view with the probe inserted in the

572 right ventrobronchus (CVB); B) the lungs in right dorsolateral view with the probe inserted in the 573 second dorsobronchus (D2). Scale bars $=1 \mathrm{~cm}$.

574

575 Fig. 2. A) Inflated lungs of a $1.01 \mathrm{~kg}$ Crocodylus niloticus (NNC3) in ventral view, head is to the right; B) ventral view of the tracheal loop and heart (pericardium has been removed) of a 10.1 $\mathrm{kg}$ C. niloticus (FNC6), head is to the right. Scale bars $=1 \mathrm{~cm}$. Arrows indicate tracheal loop; or lack thereof in the smaller individual $(A)$.

579

580 Fig. 3. 3D segmented surface models of the bronchial trees of Crocodylus niloticus

581 demonstrating the position of the caudal expansion of the caudal saccular regions of the primary

582 bronchi within the lung, all in dorsal view. A) The translucent lung surface and bronchial tree of

$583 \mathrm{NNC9}$; B) the bronchial tree of NNC9; C) the bronchial tree of NNC5; D) the bronchial tree of

584 NNC6. Abbreviations: CVB, cervical ventral bronchus; CSS, caudal sac-like structure; D2-D7, 585 dorsobronchus 2-7; Ls, lung surface; $\mathrm{Pb}$, primary bronchus. Bronchial trees are not to scale 586 relative to one another.

587

588 Fig. 4. Segmented airways and lung surface of a $0.5 \mathrm{~kg}$ specimen of Crocodylus niloticus 589 (NNC9) generated from a $\mu$ CT scan in left craniolateral view. The solid airways are visual 
590 representations of the negative spaces within the lung. A) The primary, secondary, and tertiary

591 bronchi positioned with respect to the lung surface (transparent blue); B) the primary, secondary

592 and tertiary bronchi; C) the primary and secondary bronchi. For a detailed model of the anatomy

593 see Figs. 5 and 6. Color scheme: translucent blue, lung surface; white, trachea and primary

594 bronchi; mint green, cervical ventral bronchi (CVB); lime, D2; neon green, D3; aqua, D4; light

595 aqua, D5; light blue, D6, periwinkle, D7; blue, laterobronchi; purple, caudal group bronchi

596 (CGB); red, M1; neon pink, M2; medium pink, M3; light pink, M4; pale pink, M5; pale

597 purple-deep pink-purples, M6-8; yellow-gold, cardiac lobes.

598

599 Fig. 5. Primary bronchi, ventrobronchi (CVB), dorsobronchi (D), and medial bronchi (M) of a 0.5

$600 \mathrm{~kg}$ Crocodylus niloticus (NNC9) generated from $\mu \mathrm{CT}$. The ventrobronchus and dorsobronchi in

601 A) left craniolateral view; and B) left lateral view. The ventrobronchus and medial bronchi in C)

602

right craniolateral view; and D) left lateral view. The solid airways are visual representations of

603 the negative spaces within the lung. Abbreviations: CVB, cervical ventral bronchus; D2-7,

604 dorsobronchi 2-7; M1-8, medial bronchi 1-8; Pb, primary bronchus; R, right; Tr, trachea.

605

606 Fig. 6. The primary bronchi, ventrobronchi, cardiac lobes, laterobronchi, and caudal group

607 bronchi of a $0.5 \mathrm{~kg}$ Crocodylus niloticus (NNC9) generated from $\mu \mathrm{CT}$. The lungs in A) left

608 craniolateral view; B) dorsal view; C) left lateral view; D) ventral view. The solid airways are

609 visual representations of the negative spaces within the lung. Abbreviations: C1-4, cardiac lobes

610 1-4; CGB, caudal group bronchi; CVB, cervicoventrobronchi; L, laterobronchi; Tr, trachea.

611

612 Fig. 7. Lungs of a $0.5 \mathrm{~kg}$ specimen of Crocodylus niloticus (NNC9) injected with white latex,

613 demonstrating the parabronchi $(p)$ connecting the CVB and D2. A) Lateral view of the right lung;

614 B) medial view of the sagittally-sectioned right lung stretched to expose the parabronchi

615 indicated by the pink lines; C) medial view of the sagittally-sectioned left lung. Pink arrows 
616 indicate the parabronchi. Scale bar in $A$ and $B=1 \mathrm{~cm}$; scale bar in $C=1.8 \mathrm{~mm}$. Abbreviations:

617 CVB, cervical ventral bronchus; D2-3, dorsobronchi 2-3; L, laterobronchi; P, parabronchi.

618

619 Fig. 8. Airflow in the dorsobronchi and ventrobronchi measured in excised lungs with dual

620 thermistor flow meters. A positive trace indicates that flow is caudal to cranial (black arrow); a

621 negative trace shows airflow that is cranial to caudal (white arrow). A) Direction of flow in D2

622 from NNC6; B) direction of flow at the trachea while flow was recorded in D2 in NNC6; C)

623 direction of flow in D3 from NNC6; D) direction of flow at the trachea while flow was recorded in

624 D3 in NNC6; E) direction of flow in D4 from NNC5; F) direction of flow at the trachea while flow

625 was recorded in NNC5 G) direction of flow at the trachea while flow was recorded in the CVB in

626 NNC5; H) direction of flow at the trachea while flow was recorded in the CVB in NNC5.

627

628

Fig. 9. 3D segmented models of the bronchial tree of a $0.6 \mathrm{~kg}$ specimen of Crocodylus niloticus

(NNC6) demonstrating the direction of airflow in the ventrobronchi and dorsobronchi in which

630

airflow has been directly measured during both inspiration and expiration. A) The primary,

631 secondary, and tertiary bronchi in left lateral view; the color scheme is as in Figs. 2, 6-7. B) The

632 bronchial tree in left lateral view with the left ventrobronchus (CVB) and first three dorsobronchi

633 highlighted to show direction of airflow. C) The bronchial tree in dorsal view with the

634 ventrobronchi and first three dorsobronchi highlighted to show direction of airflow. D) The

635 bronchial tree in dorsal view, with all of the secondary and tertiary bronchi removed except for

636 the secondary bronchi in which airflow was directly measured (CVB, D2-D4). E) The bronchial

637 tree in left craniolateral view with all of the secondary and tertiary bronchi removed except for

638 the secondary bronchi in which airflow was directly measured (CVB, D2-D4). Color scheme for

639 B-E: blue, airflow is cranial to caudal during both phases of ventilation; green, airflow is caudal

640 to cranial during both phases of ventilation; grey, primary bronchus.

641 
642 Fig. 10. Diagrammatic and highly simplified representation of airflow through the dorsobronchi

643 and ventrobronchi during inspiration (A) and expiration (B) in the crocodilian lung, and

644 inspiration (A) and expiration (D) in the avian lung. The avian model is a modification of the

645 Hazelhoff loop (Hazelhoff 1951). Arrows denote direction of airflow, white arrows show air

646 flowing through the parabronchi, blue arrows show air entering the trachea, the red circled " $\mathrm{X}$ "

647 demonstrates the location of the aerodynamic inspiratory valve (i.e., air does not flow through

648 this location during inspiration). Colors represent hypothesized homologous regions of the lung

649 in both groups. Abbreviations: d, dorsobronchi; P, parabronchi; Pb, primary bronchus; v, 650 ventrobronchi.

651

652 Fig. 11. 3D segmented models of the bronchial tree of two live specimens of Alligator

653 mississippiensis (in situ), and three specimens of Crocodylus niloticus generated from $\mu \mathrm{CT}$ and

654 medical grade CT, all in dorsal view. A) The primary, secondary, and tertiary bronchi of a $2.8 \mathrm{~kg}$

655

A. mississipiensis; B) the primary, secondary, and tertiary bronchi of a $11 \mathrm{~kg} \mathrm{~A}$. mississippiensis;

656

C) the primary, secondary, and tertiary bronchi of a $0.5 \mathrm{~kg} \mathrm{C.} \mathrm{niloticus} \mathrm{(NNC9);} \mathrm{D)} \mathrm{the} \mathrm{primary,}$

657 secondary, and tertiary bronchi of a $0.8 \mathrm{~kg} \mathrm{C.} \mathrm{niloticus} \mathrm{(NNC6);} \mathrm{E)} \mathrm{the} \mathrm{primary,} \mathrm{secondary,} \mathrm{and}$

658 tertiary bronchi of a $0.9 \mathrm{~kg}$ C. niloticus (NNC5). Images not to scale. Color scheme: white, trachea and primary bronchi; mint green, cervicoventrobronchi (CVB); lime, D2; neon green, D3; aqua, D4; light aqua, D5; light blue, D6, periwinkle, D7; blue, laterobronchi; purple, caudal group

661 bronchi (CGB); red, M1; neon pink, M2; medium pink, M3; light pink, M4; pale pink, M5; pale 662 purple-deep pink-purples, M6-8; yellow-gold, cardiac lobes.

663

664 Fig. 12. 3D segmented models of the bronchial tree of two live specimens of $A$. mississippiensis 665 (in situ) and three cadaveric specimens of Crocodylus niloticus generated from $\mu \mathrm{CT}$ and 666 medical grade CT, all in left lateral view. A) The primary, secondary, and tertiary bronchi of a 667 2.8kg A. mississippiensis; B) the primary, secondary, and tertiary bronchi of a $11 \mathrm{~kg} \mathrm{A.}$ 
668 mississippiensis; C) the primary, secondary, and tertiary bronchi of a $0.5 \mathrm{~kg}$ C. niloticus (NNC9);

669 D) the primary, secondary, and tertiary bronchi of a $0.8 \mathrm{~kg} \mathrm{C}$. niloticus (NNC6); E) the primary,

670 secondary, and tertiary bronchi of a $0.9 \mathrm{~kg} \mathrm{C}$. niloticus (NNC5). Images not to scale. Color

671 scheme: white, trachea and primary bronchi; mint green, cervicoventrobronchi (CVB); lime, D2;

672 neon green, D3; aqua, D4; light aqua, D5; light blue, D6, periwinkle, D7; blue, laterobronchi;

673 purple, caudal group bronchi (CGB); red, M1; neon pink, M2; medium pink, M3; light pink, M4;

674 pale pink, M5; pale purple-deep pink-purples, M6-8; yellow-gold, cardiac lobes.

675

676 Fig. 13. Diagrammatic representations of the crocodilian (A) and avian (B) lungs in left lateral

677 view with colors identifying proposed homologous characters within the bronchial tree and air

678 sac system of both groups. The image of the bird is modified from Duncker (1971).

679 Abbreviations: AAS, abdominal air sac; CAS, cervical air sac; CRTS, cranial thoracic air sac;

680

CSS, caudal sac-like structure; CTS, caudal thoracic air sac; d, dorsobronchi; GL,

681

gas-exchanging lung; HS, horizontal septum; L, laterobronchi; NGL, non-gas-exchanging lung;

682 ObS, oblique septum; P, parabronchi; Pb, primary bronchus; $\mathrm{Tr}$, trachea; $\mathrm{v}$, ventrobronchi.

683

684

685

\section{References}

686

687 Berner RA, VandenBrooks JM, Ward PD. 2007. Oxygen and evolution. Science 316:

688 557-558.

689

690

691

692

693 Boelert R. 1942. Sur la physiologie de la respirataion de l' Alligator mississippiensis.

694 Arch. Int. Physiol. 52: 57-72.

695

696 Bonde N and Christiansen P. 2003. The detailed anatomy of Rhamphorhynchus: axial 697 pneumaticity and its implications. London, Geological Society. 698 
699

700

701

702

703

704

705

706

707

708

709

710

711

712

713

714

715

716

717

718

719

720

721

722

723

724

725

726

727

728

729

730

731

732

733

734

735

736

737

738

739

740

741

742

743

744

Benson RBJ, Butler RJ, Carrano MT, O'Connor PM. 2011. Air-filled postcranial bones in theropod dinosaurs: physiological implications and the 'reptile'-bird transition. Biological Reviews 87:168-193.

Brackenbury JH. 1971. Airflow dynamics in the avian lung as determined by direct and indirect methods. Respiration Physiology 13: 318-329.

Brackenbury JH. 1972. Physical determinants of airflow pattern within the avian lung. Respiration Physiology 15: 384-397.

Brackenbury JH. 1979. Corrections to the Hazelhoff model of airflow in the avian lung. Respiration Physiology 36: 143-154.

Brackenbury JH. 1987. Ventilation of the lung-air sac system. Bird Respiration. Seller TJ. Boca Raton, CRC Press. II: 39-69.

Brochu CA. 1997. Morphology, fossils, divergence timing, and the phylogenetic relationships of Gavialis. Systemiatic Biology 46: 479-522.

Broman I. 1939. Die Embryonalentwicklung der Lungen bei Krokodilen und Seesschildkröten. Jb. morphol. mikrosk. Anat. 84: 224-306.

Brown RE, Brain JD, Wang N. 1997. The avian respiratory system: a unique model for studies of respiratory toxicosis and for monitoring air quality. Environmental Health Perspectives 105:188-200.

Brusatte SL, Benton MJ, Graeme TL, Ruta M and Wang SC. 2010. Marcroevolutionary patterns in the evolutionary radiation of archosaurs (Tetrapoda: Diapsida). Earth and Environmental Science Transactions of the Royal Society of Edenburgh 101: 367-382.

Butler JP, Banzett RB and Fredberg JJ. 1988. Inspiratory valving in avian bronchi: aerodynamic considerations. Respiration Physiology 72: 241-256.

Butler RJ, Barrett PM and Gower DJ. 2012. Reassessment of the evidence for postcranial skeletal pneumaticity in Triassic archosaurs, and the early evolution of the avian respiratory system. PLOS ONE 7: e34094.

Claessens LPAM, O'Connor PMO and Unwin DM. 2009. Respiratory evolution facilitated the origin of pterosaur flight and aerial gigantism. PLOS one 4(2): e4497. doi:4410.1371/journal.pone.0004497.

Cuvier G. 1840. Leçons d'Anatomie Comparée, Rédigées et Publiées par Duvernoy. Paris.

Dejours P. 1981. Principles of Comparative Respiratory Physiology. Elsevier/North-Holland Biomedical Press. Amsterdam. 
752

753

754

755

756

757

758

759

760

761

762

763

764

765

766

767

768

769

770

771

772

773

774

775

776

777

778

779

780

781

782

783

784

785

786

787

788

789

Dotterweich H. 1936. Die Atmung der Vögel. Z.f. vergl. Physiol. 23: 744-770.

Duncker HR. 1971. Advances in Anatomy and Embryology and Cell Biology: The Air Sac System of Birds. New York, Springer-Verlag.

Duncker HR. 1971. The lung air sac system of birds. A contribution to the functional anatomy of the respiratory apparatus. Ergebnisse der Anatomie und Entwicklungsgeschichte 45: 1-171.

Duncker HR. 1972. Structure of avian lungs. Respiration Physiology 14: 44-63.

Duncker HR. 1978. General morphological principles of amnioteic lungs. Respiratory function in birds. Piiper J. Heidelberg, Springer-Verlag.

Farmer CG. 2006. On the origin of avian air sacs. Respiration Physiology and Neurobiology 154: 89-106.

Farmer CG. 2010. The provenance of alveolar and parabronchial lungs: insights from paleoecology and the discovery of cardiogenic, unidirectional airflow in the American alligator (Alligator mississippiensis). Physiological and Biochemical Zoology 83(4): 561-575.

Farmer CG and Sanders K. 2010. Unidirectional airflow in the lungs of alligators. Science 327(5963): 338-340.

Gatesy J, Amato G, Norell M, DeSalle R and Hayashi C. 2003. Combined support for wholesale taxic atavism in gavialine crocodylians. Systemiatic Biology 52: 403-422.

Graham JB, Dudley R, Aguilar NM, Gans C. 1995. Implications of the late Palaeozoic oxygen pulse for physiology and evolution. Nature 375:117-120.

Hazelhoff EH. 1951. Structure and function of the lung of birds. Poultry Science 30: 3-10.

Huey RB, Ward PD. 2005. Hypoxia, global warming and terrestrial Late Permain extinctions. Science 308: 398-401.

Huxley TH. 1882. On the respiratory organs of Apteryx. Proceedings of the Zoological Society of London 1882: 560-569.

Jeffery NS, Stephenson RS, Gallagher JA, Jarvis JC and Cox PG. 2011.

Micro-computed tomography with iodine staining resolves the arrangement of muscle fibres. Journal of Biomechanics 44: 189-192. 
790

791

792

793

794

795

796

797

798

799

800

801

802

803

804

805

806

807

808

809

810

811

812

813

814

815

816

817

818

819

820

821

822

823

824

825

826

827

828

829

830

831

832

833

834

835

Kaiser A, Klok CJ, Socha JJ, Lee WK, Quinlan MC, Harrison JF. 2007. Increase in tracheal investment with beetle size supports hypothesis of oxygen limitation on insect gigantism. Proceedings of the National Academy of Sciences 104:13198-13203.

King AS, Ed. 1966. Structural and functional aspects of the avian lungs and air sacs. International Review of General and Experimental Zoology. New York and London, Academic Press.

Klemm RD, Gatz RN, Westfall JA and Fedde MR. 1979. Microanatomy of the lung parenchyma of a teju lizard Tupinambis nigropunctatus. Journal of Morphology 161: 257-280.

Lereboullet A. 1838. Anatomie Comparée de l'Appareil Respiratoire dans les Animaux Vertébrés. Paris.

Locy WA and Larsell O. 1916. The embryology of the bird's lung based on observations of the domestic fowl Part I. The American Journal of Anatomy 19(3): 447-506.

Maina J and Nathaniel C. 2001. A qualitative and quantitative study of the lung of an ostrich. Journal of Experimental Biology 204: 2313-2330.

Maina JN. 2000. What it takes to fly: the structural and functional respiratory refinements in birds and bats. Journal of Experimental Biology 203: 3045-3064.

Maina JN. 2006. Development, structure, and function of a novel respiratory organ, the lung-air sac system of birds: to go where no other vertebrate has gone. Biological Reviews 81: 545-579.

Maina JN, West JB. 2005. Thin and strong! The bioengineering dilemma in the structural and functional design of the blood-gas barrier. Physiological Reviews 95: 811-844.

Metzger RJ, Klein OD, Martin GR and Krasnow MA. 2008. The branching programme of mouse lung development. Nature 453: 745-751.

Meyer M, Scheid P and Piiper J. 1981. Comparison birds/mammals: structure and function of the gas exchange apparatus. Respiration: proceedings of the 28th international congress of physiological sciences, Budapest, 1980. (Advances in Physiological Sciences). Hutas I and Debreczeni A. Oxford, England, Pergamon Press. 10 Respiration: 135-143.

Milani A. 1894. Beiträge zur Kenntniss der Reptilienlunge. . Zoologische Jahrbücher 7: 545-592.

Milani A. 1897. Beiträge zur Kenntnis der Reptilienlunge. II. Zoologische Jahrbücher 10: 93-156. 
836

837

838

839

840

841

842

843

844

845

846

847

848

849

850

851

852

853

854

855

856

857

858

859

860

861

862

863

864

865

866

867

868

869

870

871

872

873

874

875

876

877

878

879

880

Moser F. 1902. Beiträge zur vergleichenden Entwicklungsgeschichte der

Wirbeltierlunge. Arch mikrosk Anat Entwicklungsmech 60: 587-668.

Mushonga B and Horowitz A. 1996. Serous cavities of the Nile crocodile (Crocodylus niloticus). Journal of Zoo and Wildlife Medicine 27(2): 170-179.

Nesbitt SJ. 2011. The early evolution of archosaurs: relationships and the origin of major clades'. Bulletin American Museum of Natural History 352: 1-292.

Nysten M. 1962. Étude anatomique des rapports de la vessie aérienne avec l'axe vertébral chez Pantadon buchholzi Peters. Annales Musée Royal de l'Afrique Centrale 8:187-220.

O'Connor PM. 2009. Evolution of the archosaurian body plans: skeletal adaptations of an air-sac-based breathing apparatus in birds and other archosaurs. Journal of Experimental Zoology 311A: 629-646.

O'Connor PMO and Claessens LPAM. 2005. Basic avian pulmonary design and flow-through ventilation in non-avian theropod dinosaurs. Nature 436: 253-256.

Oaks JR. 2011. A time-calibrated species tree of Crocodylia reveals a recent radiation of the true crocodiles. Evolution 65: 3285-3297.

Perry SF. 1988. Functional morphology of the lungs of the nile crocodile, Crocodylus niloticus: non-respiratory parameters. Journal of Experimental Biology 134: 99-117.

Perry SF. 1989. Structure and function of the reptilian respiratory system. Comparative Pulmonary Physiology. Current Concepts. Wood SC. New York, Marcel Dekker Inc.: 193-236.

Perry SF. 1990. Gas exchange strategy in the Nile crocodile: a morphometric study. Journal of Comparative Physiology B 159: 761-769.

Perry SF. 1992. Gas exchange strategies of reptiles and the origin of the avian lung. Physiological adaptations in vertebrates; respiration, circulation, and metabolism. Wood S, Weber R, Hargens A and Millard R. New York, Marcel Dekker, Inc. 56: 149-167.

Perry SF. 1998. Lungs: Comparative anatomy, functional morphology, and evolution. Biology of the Reptilia. Gans C and Gaunt AS. Ithaca, New York, Society for the Study of Amphibians and Reptiles. 19 (Morphology G): 1-92.

Perry SF. 2001. Functional morphology of the reptilian and avian respiratory systems and its implications for theropod dinosaurs. New Perspectives on the Origin and Early Evolution of Birds: Proceedings of the International Symposium in Honor of John H. 
881 Ostrom. Gauthier JA and Gall LF. New Haven, Peabody Museum of Natural History,

882 Yale University: 429-441.

883

884 Perry SF and Duncker HR. 1978. Lung architecture, volume and static mechanics in five 885 species of lizards. Respiration Physiology 34: 61-81.

886

912

Perry SF, Breuer T, Pajor N. 2011. Structure and function of the sauropod respiratory system. In: Klein N, Remes K, Gee CT, Sander PM, eds. Biology of the sauropod dinosaurs: Understanding the life of giants. Bloomington \& Indianapolis: Indiana University Press. pp 83-93.

Plummer EM and Goller F. 2008. Singing with reduced air sac volume causes uniform decrease in airflow and sound amplitude in the zebra finch. Journal of Experimental Biology 211: 66-78.

Reese AM. 1915. The Alligator and Its Allies. New York, G.P. Putnam's Sons.

Sanders RK and Farmer CG. 2012. The pulmonary anatomy of Alligator mississippiensis and its similarity to the avian respiratory system. The Anatomical Record 295: 699-714.

Sappey P-C. 1847. Recherches sur L'appareil Respiratoire des Oiseaux. Pairs, Germer-Bailliere.

Schachner ER, Lyson TR, Dodson P. 2009. Evolution of the respiratory system in nonavian theropods: evidence from rib and vertebral morphology. The Anatomical Record 292:1501-1513.

Schachner ER, Farmer CG, McDonald AT, Dodson P. 2011. Evolution of the dinosauriform respiratory apparatus: new evidence from the postcranial axial skeleton. The Anatomical Record 294:1531-1547.

Sereno PC, Martinez RN, Wilson JA, Varricchio DJ, Alcober OA and Larsson HCE. 2008. Evidence for avian intrathoracic air sacs in a new predatory dinosaur from Argentina. PLOS ONE 3(9): e3303.

Wallach V. The lungs of snakes. In: Gans C, Gaunt AS, editors. Biology of the Reptilia: visceral organs. New York: Society for the Study of Amphibians and reptiles. p 93-295.

Wang N, Banzett RB, Butler JP and Fredberg JJ. 1988. Bird lung models show that convective inertia effects inspiratory aerodynamic valving. Respiration Physiology 73: 111-124.

Wedel MJ. 2003. Vertebral pneumaticity, air sacs, and the physiology of sauropod dinosaurs. Paleobiology 29:243-255. 
927 Wedel MJ. 2006. Origin of postcranial skeletal pneumaticity in dinosaurs. Integrative 928 Zoology 2:80-85.

929

930 Wedel M. 2007. What pneumaticity tells us about 'prosauropods', and vice versa.

931 Special Papers in Palaeontology 77: 207-222.

932

933 Wedel MJ. 2009. Evidence for bird-like air sacs in saurischian dinosaurs. Journal of 934 Experimental Zoology 311A: 611-628.

935

936 Witmer LM. 1997. The evolution of the antorbital cavity of archosaurs: a study in

937 soft-tissue reconstruction in the fossil record with an analysis of the function of

938 pneumaticity. Journal of Vertebrate Paleontology, 17(S1): 1-76.

939

940

941

942

Yates AM, Wedel MJ and Bonnan MF. 2012. The early evolution of postcranial skeletal pneumaticity in sauropodomorph dinosaurs. Acta Palaeontologica Polonica 57: 85-100. 


\section{Figure 1}

Figure 1

Excised lungs from a $3.1 \mathrm{~kg}$ Nile crocodile (NNC1; Crocodylus niloticus) demonstrating probe placement, head is to the right. A) Lungs in ventral view with the probe inserted in the right ventrobronchus (CVB); B) the lungs in right dorsolateral view with the probe inserted in the second dorsobronchus (D2). Scale bars $=1 \mathrm{~cm}$. 


\section{Figure 2}

Figure 2

A) Inflated lungs of a $1.01 \mathrm{~kg}$ Crocodylus niloticus (NNC3) in ventral view, head is to the right; B) ventral view of the tracheal loop and heart (pericardium has been removed) of a $10.1 \mathrm{~kg}$ C. niloticus (FNC6), head is to the right. Scale bars $=1 \mathrm{~cm}$. Arrows indicate tracheal loop; or lack thereof in the smaller individual (A). 


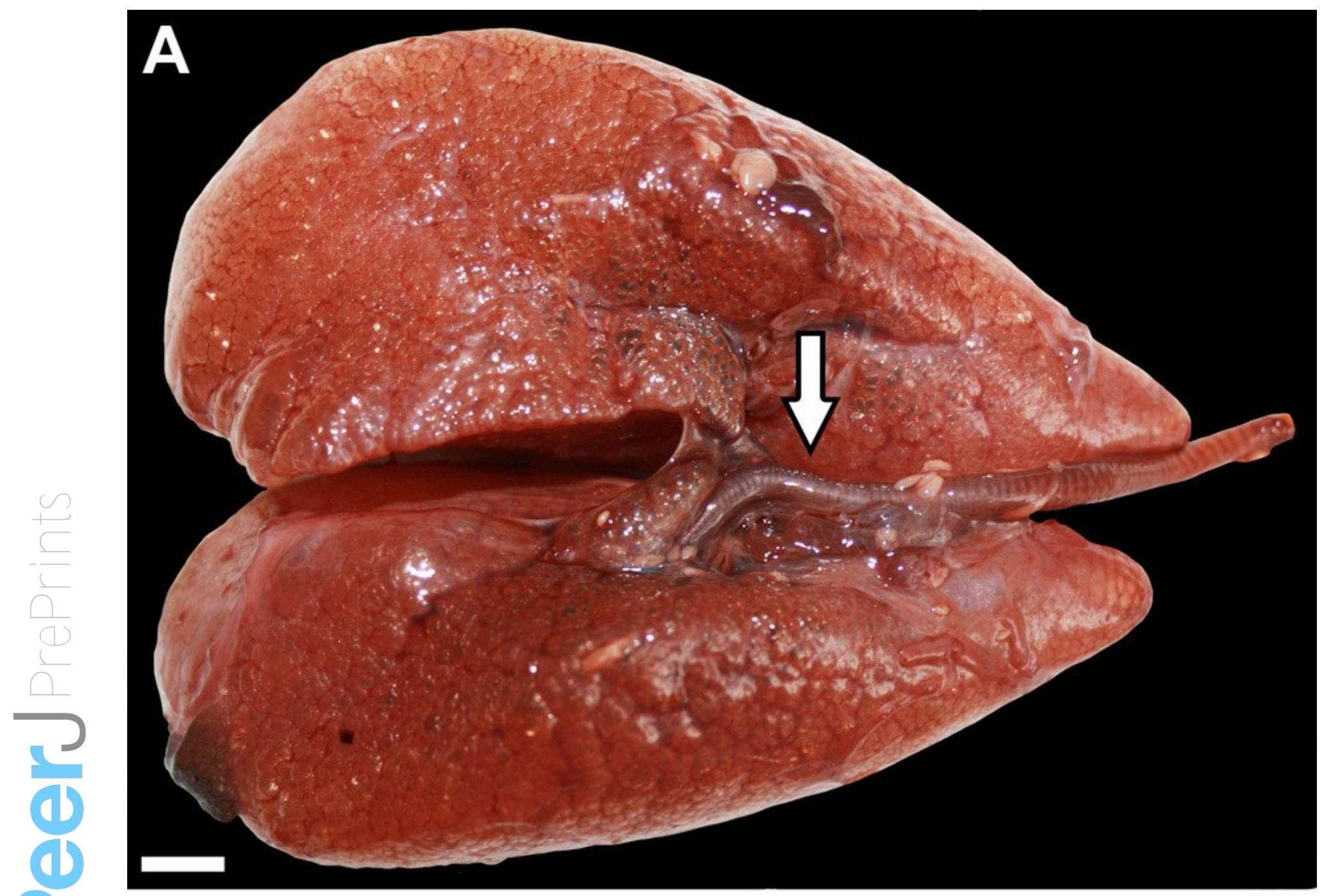

,

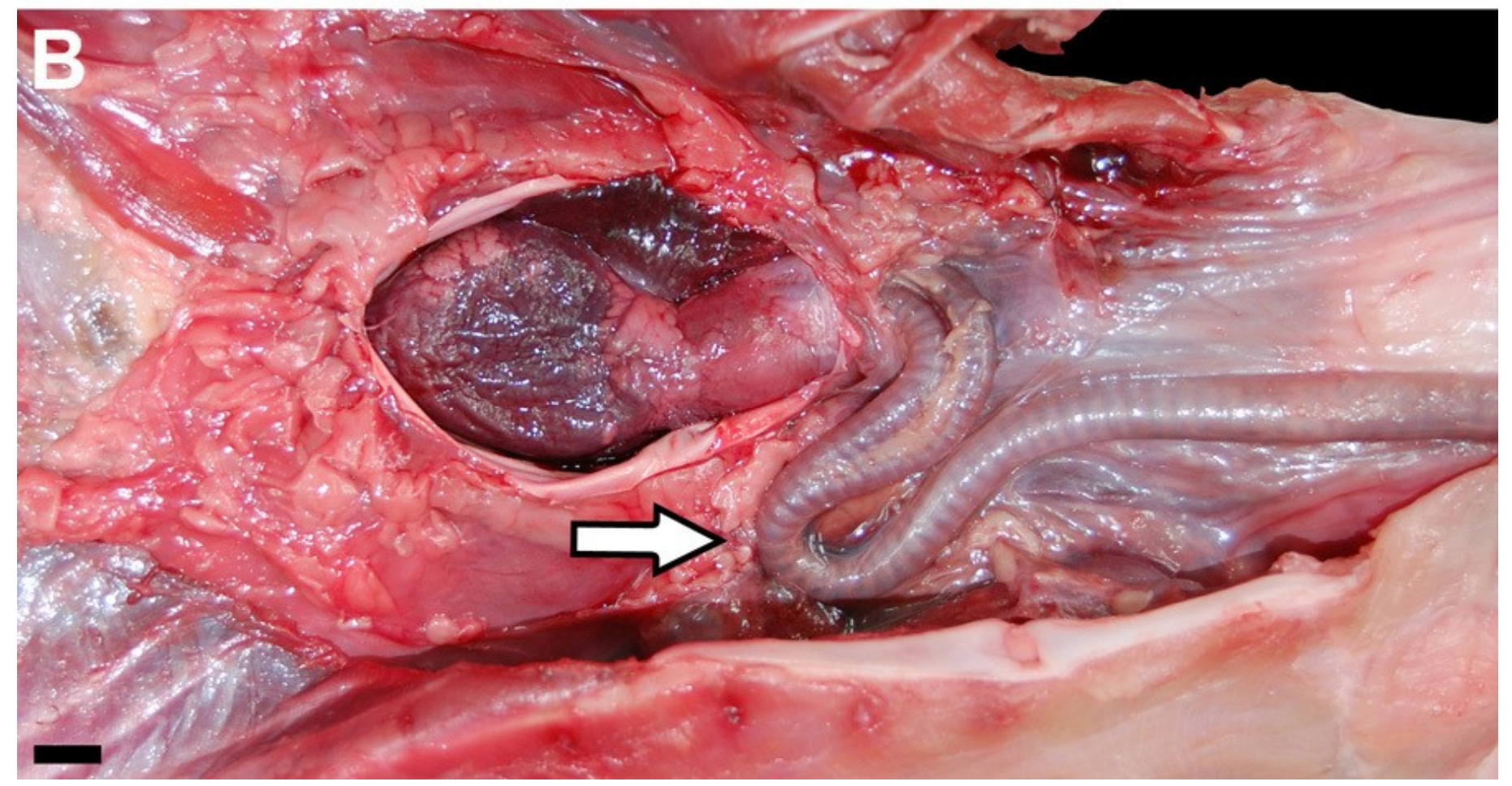




\section{Figure 3}

Figure 3

3D segmented surface models of the bronchial trees of Crocodylus niloticus demonstrating the position of the caudal expansion of the caudal saccular regions of the primary bronchi within the lung, all in dorsal view. A) The translucent lung surface and bronchial tree of NNC9; B) the bronchial tree of NNC9; C) the bronchial tree of NNC5; D) the bronchial tree of NNC6. Abbreviations: CVB, cervical ventral bronchus; CSS, caudal sac-like structure; D2-D7, dorsobronchus 2-7; Ls, lung surface; Pb, primary bronchus. Bronchial trees are not to scale relative to one another. 

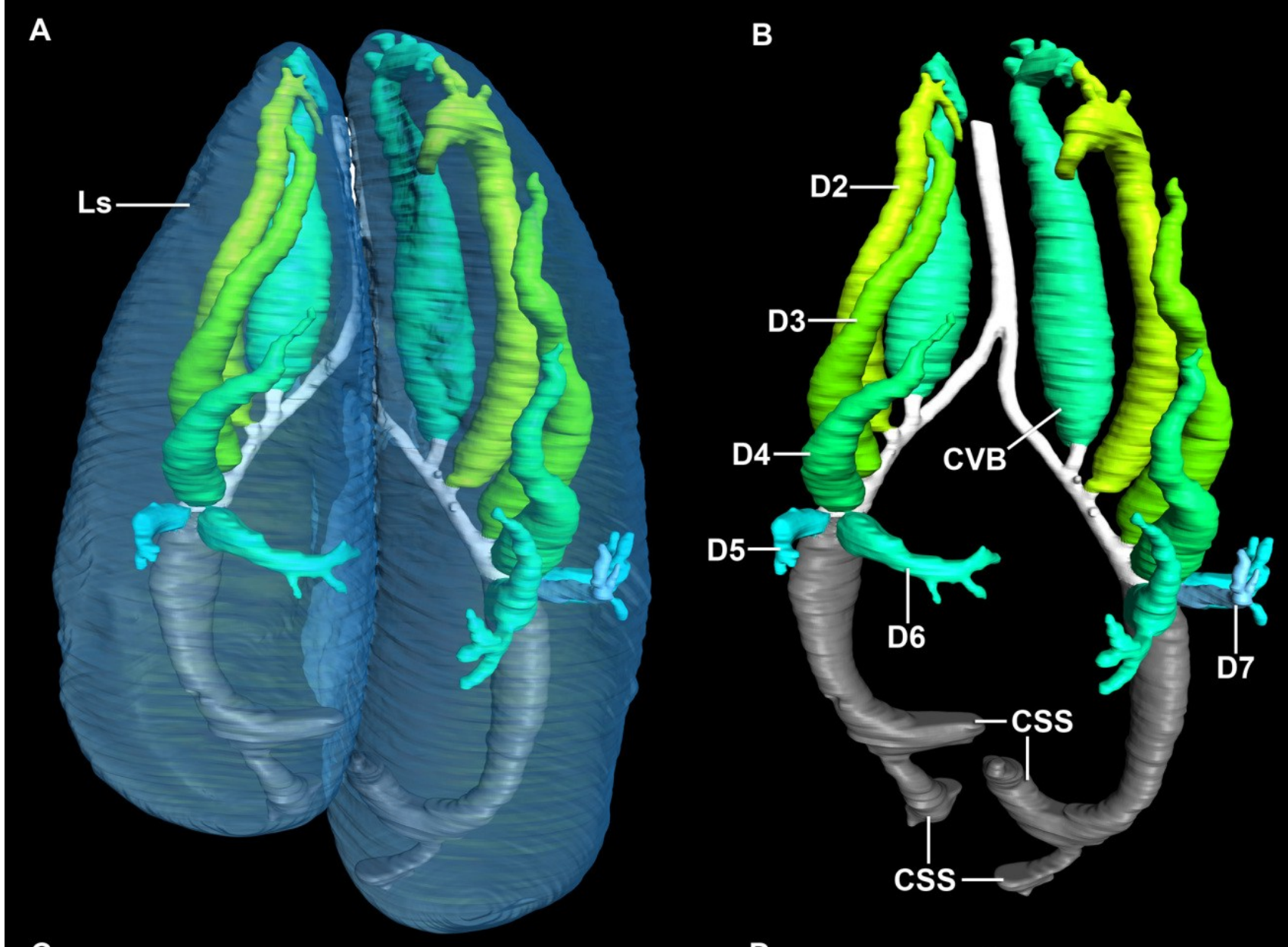

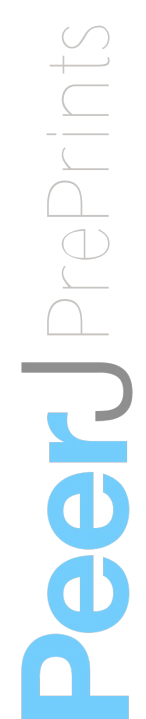
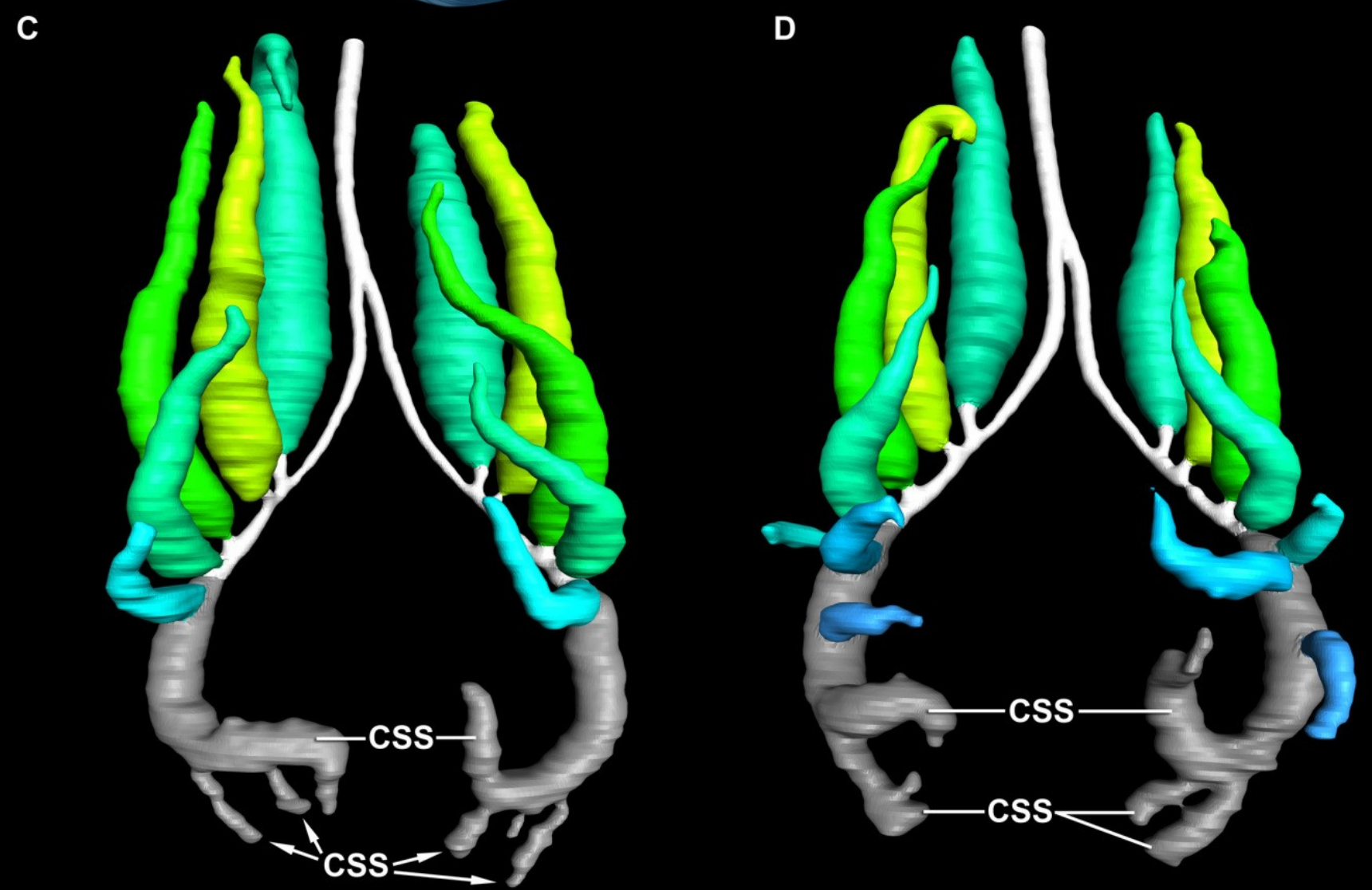


\section{Figure 4}

Figure 4

egmented airways and lung surface of a $0.5 \mathrm{~kg}$ specimen of Crocodylus niloticus (NNC9) generated from a $\mu \mathrm{CT}$ scan in left craniolateral view. The solid airways are visual representations of the negative spaces within the lung. A) The primary, secondary, and tertiary bronchi positioned with respect to the lung surface (transparent blue); B) the primary, secondary and tertiary bronchi; C) the primary and secondary bronchi. For a detailed model of the anatomy see Figs. 5 and 6. Color scheme: translucent blue, lung surface; white, trachea and primary bronchi; mint green, cervical ventral bronchi (CVB); lime, D2; neon green, D3; aqua, D4; light aqua, D5; light blue, D6, periwinkle, D7; blue, laterobronchi; purple, caudal group bronchi (CGB); red, M1; neon pink, M2; medium pink, M3; light pink, M4; pale pink, M5; pale purple-deep pink-purples, M6-8; yellow-gold, cardiac lobes. 


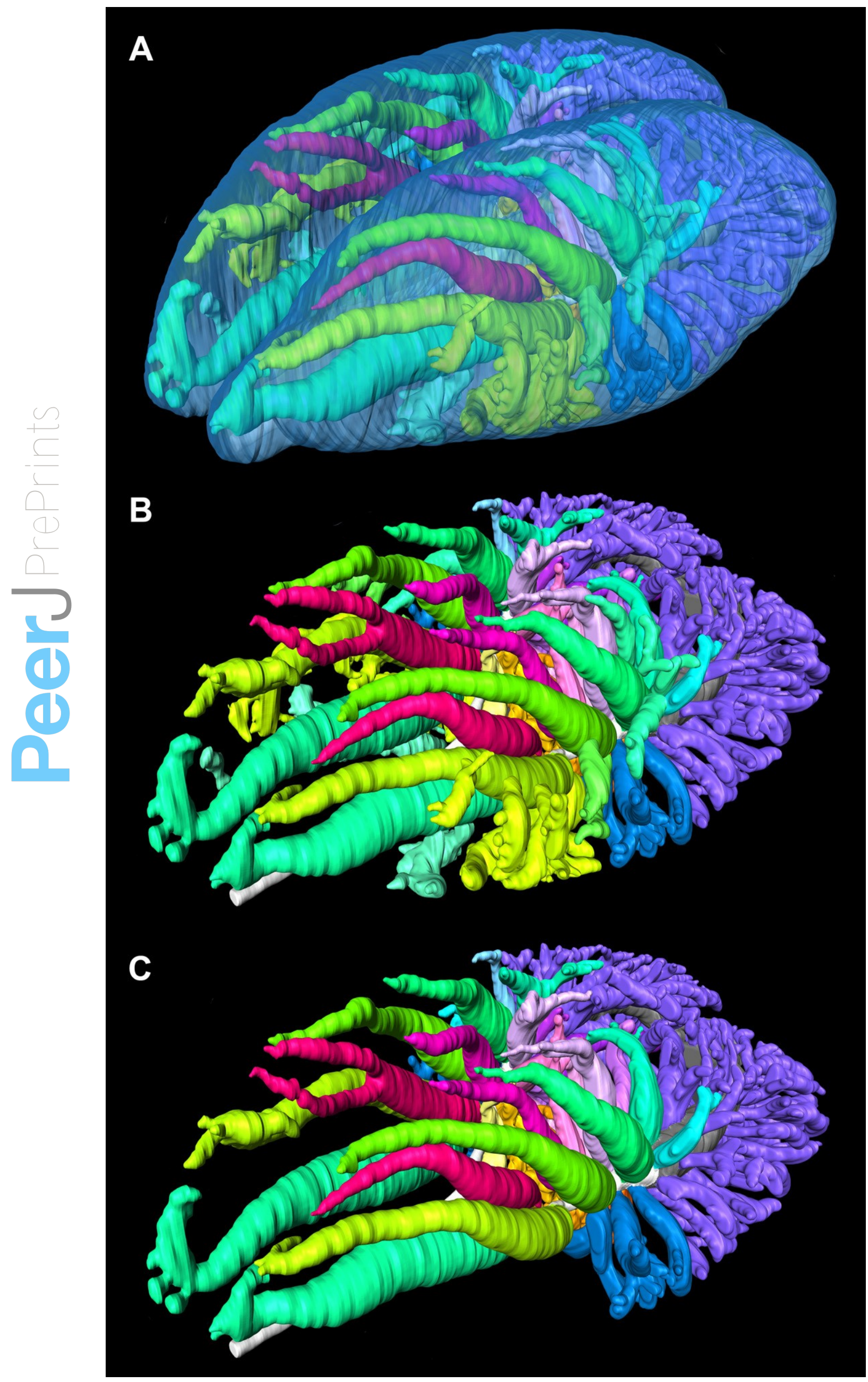




\section{Figure 5}

Figure 5

Primary bronchi, ventrobronchi (CVB), dorsobronchi (D), and medial bronchi (M) of a $0.5 \mathrm{~kg}$

Crocodylus niloticus (NNC9) generated from $\mu \mathrm{CT}$. The ventrobronchus and dorsobronchi in A) left craniolateral view; and B) left lateral view. The ventrobronchus and medial bronchi in C) right craniolateral view; and D) left lateral view. The solid airways are visual representations of the negative spaces within the lung. Abbreviations: CVB, cervical ventral bronchus; D2-7, dorsobronchi 2-7; M1-8, medial bronchi 1-8; Pb, primary bronchus; $\mathrm{R}$, right; $\mathrm{Tr}$, trachea.

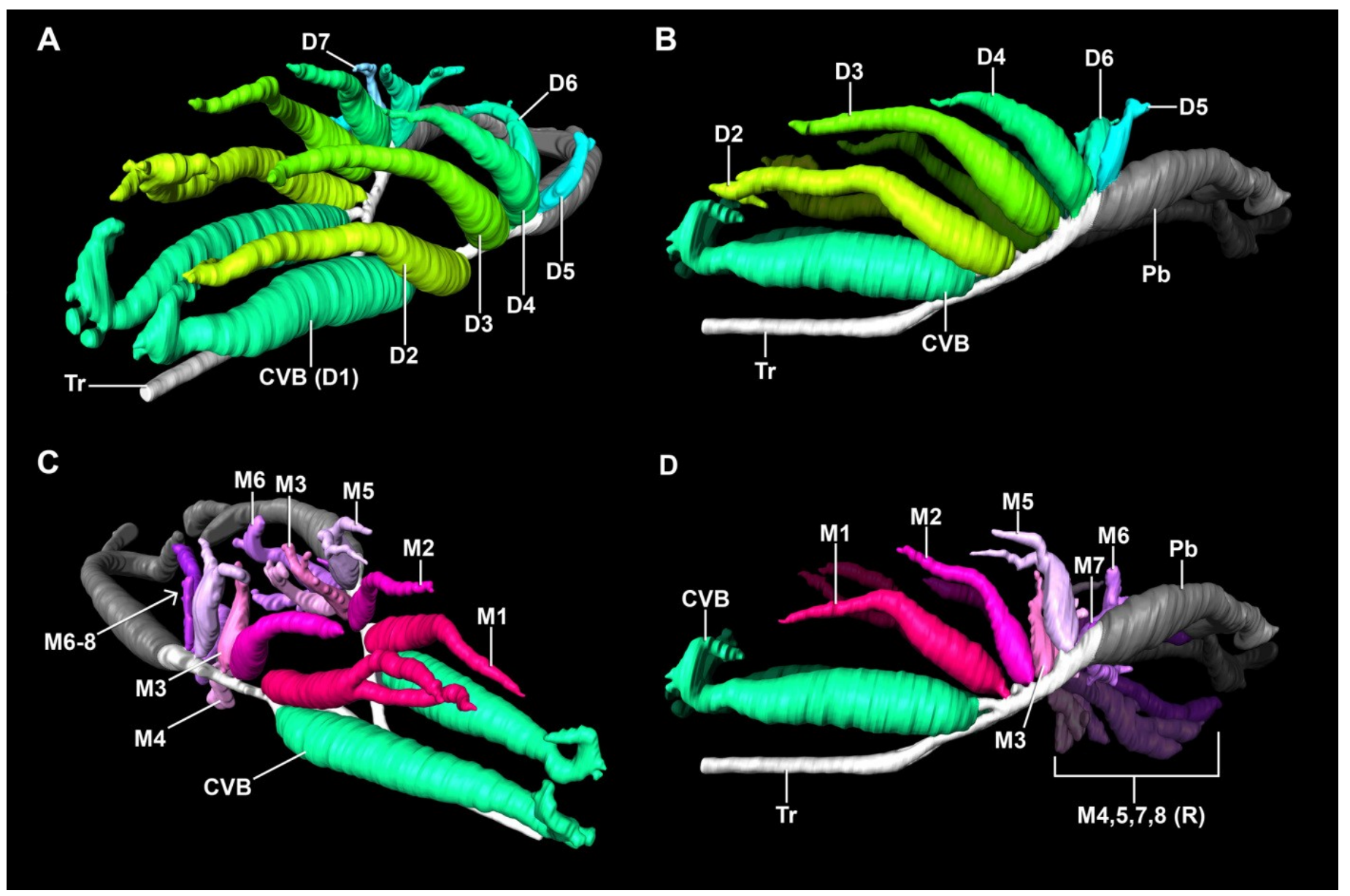




\section{Figure 6}

Figure 6

The primary bronchi, ventrobronchi, cardiac lobes, laterobronchi, and caudal group bronchi of a $0.5 \mathrm{~kg}$ Crocodylus niloticus (NNC9) generated from $\mu \mathrm{CT}$. The lungs in A) left craniolateral view; B) dorsal view; C) left lateral view; D) ventral view. The solid airways are visual representations of the negative spaces within the lung. Abbreviations: C1-4, cardiac lobes 1-4; CGB, caudal group bronchi; CVB, cervicoventrobronchi; L, laterobronchi; Tr, trachea.

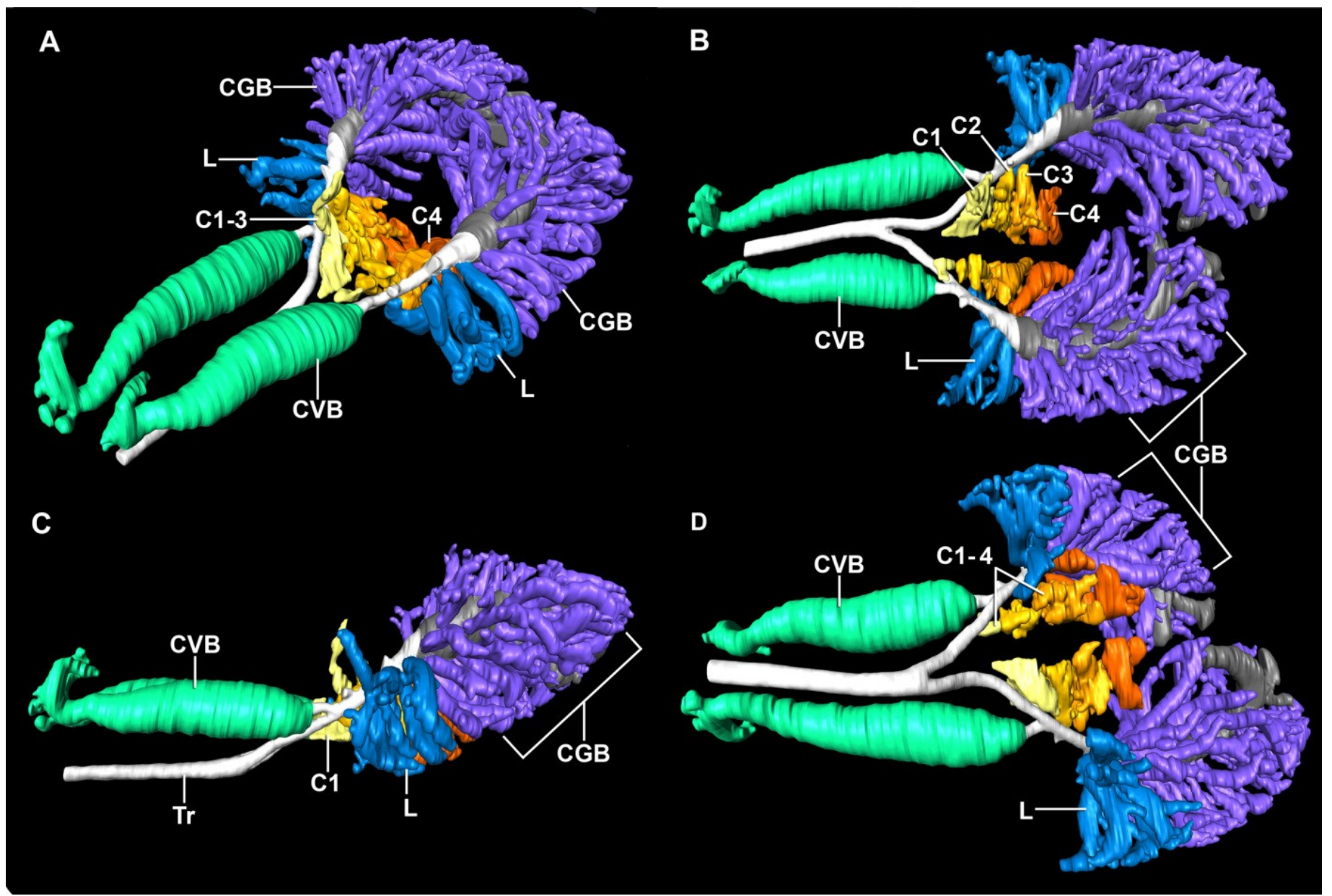




\section{Figure 7}

Figure 7

Lungs of a $0.5 \mathrm{~kg}$ specimen of Crocodylus niloticus (NNC9) injected with white latex, demonstrating the parabronchi (p) connecting the CVB and D2. A) Lateral view of the right lung; B) medial view of the sagittally-sectioned right lung stretched to expose the parabronchi indicated by the pink lines; C) medial view of the sagittally-sectioned left lung. Pink arrows indicate the parabronchi. Scale bar in A and $\mathrm{B}=1 \mathrm{~cm}$; scale bar in $\mathrm{C}=1.8 \mathrm{~mm}$. Abbreviations: CVB, cervical ventral bronchus; D2-3, dorsobronchi 2-3; L, laterobronchi; P, parabronchi.
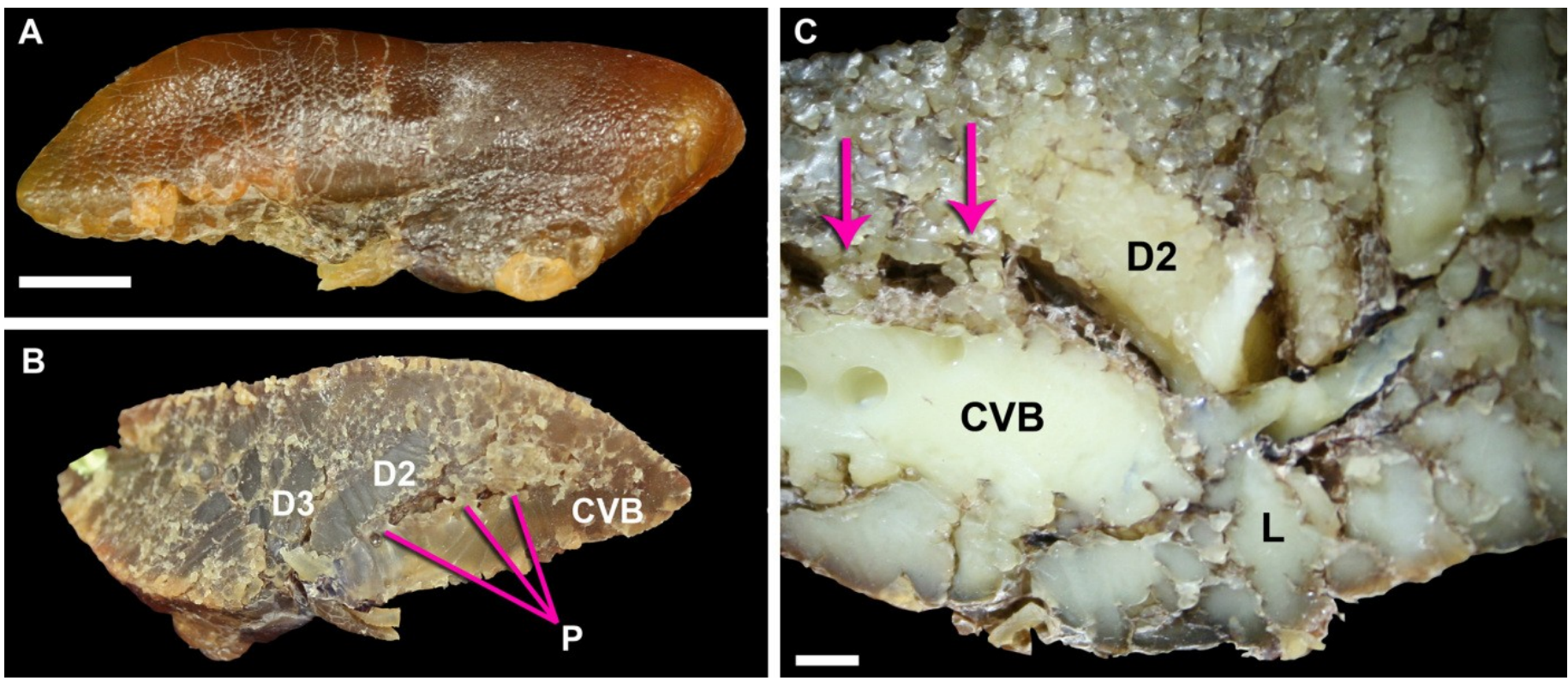


\section{Figure 8}

Figure 8

Airflow in the dorsobronchi and ventrobronchi measured in excised lungs with dual thermistor flow meters. A positive trace indicates that flow is caudal to cranial (black arrow); a negative trace shows airflow that is cranial to caudal (white arrow). A) Direction of flow in D2 from NNC6; B) direction of flow at the trachea while flow was recorded in D2 in NNC6; C) direction of flow in D3 from NNC6; D) direction of flow at the trachea while flow was recorded in D3 in NNC6; E) direction of flow in D4 from NNC5; F) direction of flow at the trachea while flow was recorded in NNC5 G) direction of flow at the trachea while flow was recorded in the CVB in NNC5; H) direction of flow at the trachea while flow was recorded in the CVB in NNC5. 


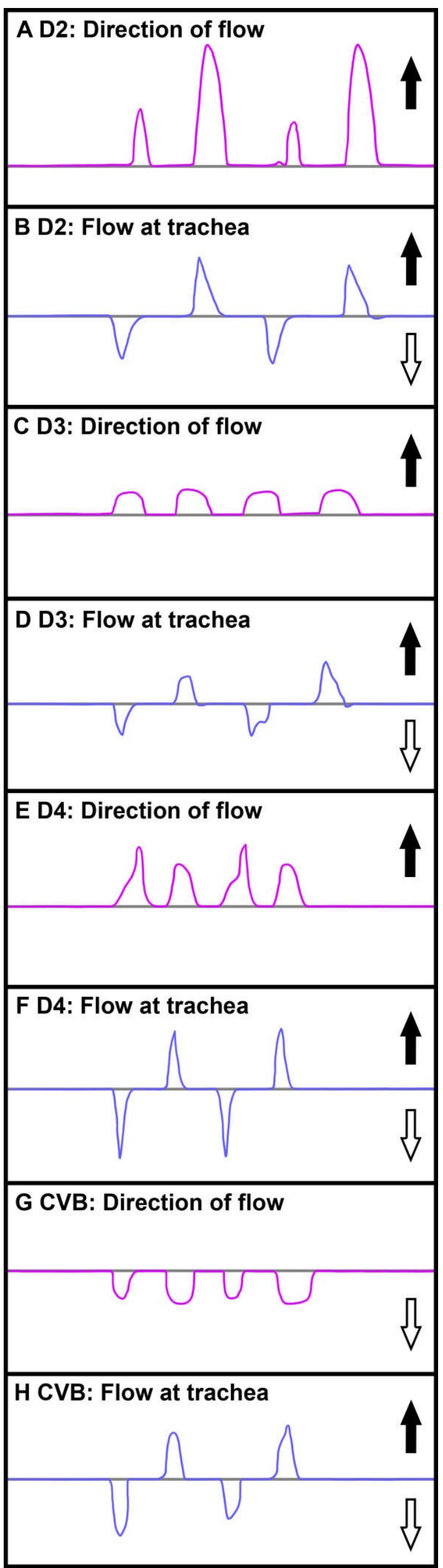




\section{Figure 9}

Figure 9

3D segmented models of the bronchial tree of a $0.6 \mathrm{~kg}$ specimen of Crocodylus niloticus (NNC6) demonstrating the direction of airflow in the ventrobronchi and dorsobronchi in which airflow has been directly measured during both inspiration and expiration. A) The primary, secondary, and tertiary bronchi in left lateral view; the color scheme is as in Figs. 2, 6-7. B) The bronchial tree in left lateral view with the left ventrobronchus (CVB) and first three dorsobronchi highlighted to show direction of airflow. C) The bronchial tree in dorsal view with the ventrobronchi and first three dorsobronchi highlighted to show direction of airflow. D) The bronchial tree in dorsal view, with all of the secondary and tertiary bronchi removed except for the secondary bronchi in which airflow was directly measured (CVB, D2-D4). E) The bronchial tree in left craniolateral view with all of the secondary and tertiary bronchi removed except for the secondary bronchi in which airflow was directly measured (CVB, D2-D4). Color scheme for B-E: blue, airflow is cranial to caudal during both phases of ventilation; green, airflow is caudal to cranial during both phases of ventilation; grey, primary bronchus. 


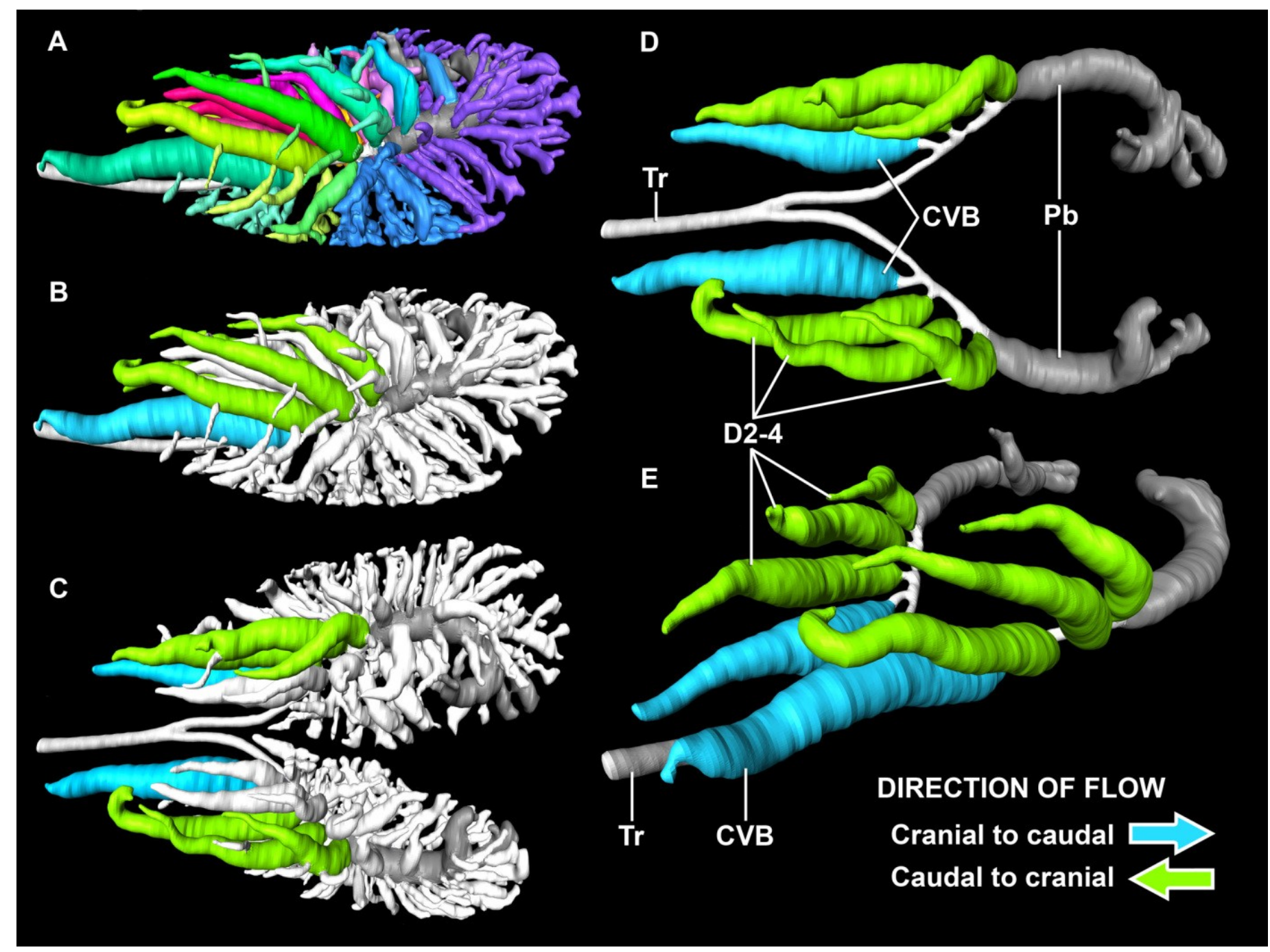




\section{Figure 10}

Figure 10

Diagrammatic and highly simplified representation of airflow through the dorsobronchi and ventrobronchi during inspiration (A) and expiration (B) in the crocodilian lung, and inspiration (A) and expiration (D) in the avian lung. The avian model is a modification of the Hazelhoff loop ( Hazelhoff 1951 ). Arrows denote direction of airflow, white arrows show air flowing through the parabronchi, blue arrows show air entering the trachea, the red circled " $\mathrm{X}$ " demonstrates the location of the aerodynamic inspiratory valve (i.e., air does not flow through this location during inspiration). Colors represent hypothesized homologous regions of the lung in both groups. Abbreviations: d, dorsobronchi; P, parabronchi; Pb, primary bronchus; v, ventrobronchi.

A

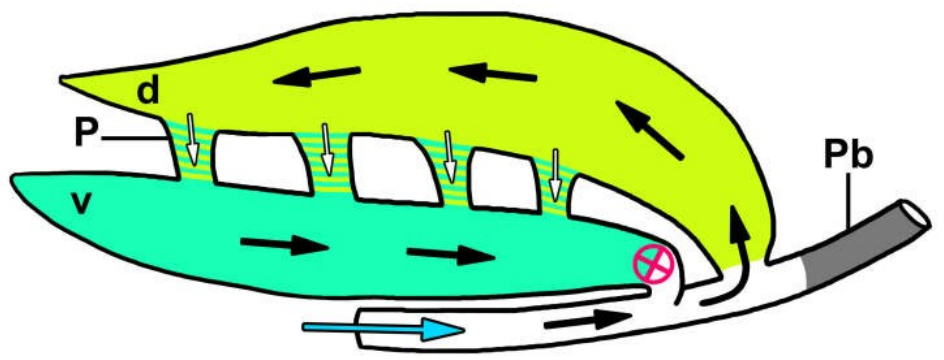

B

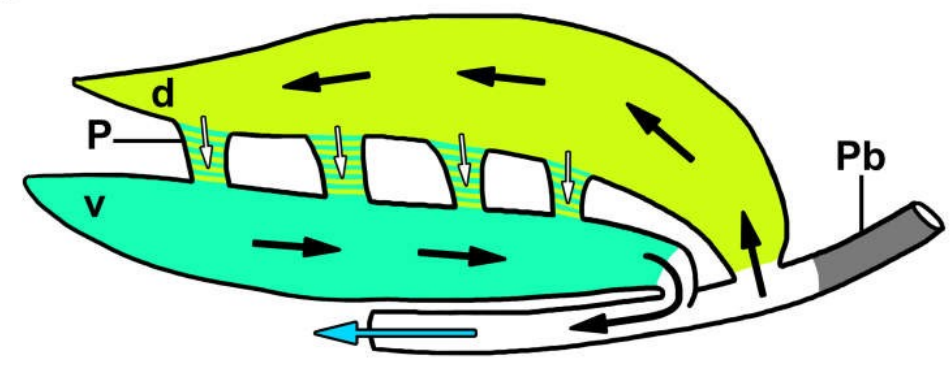

C

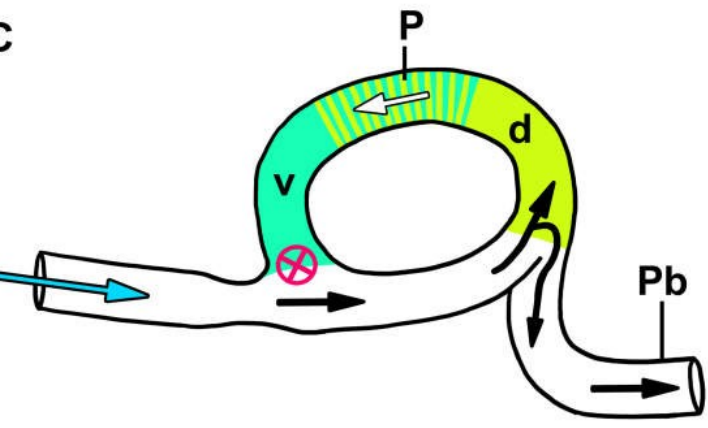

D

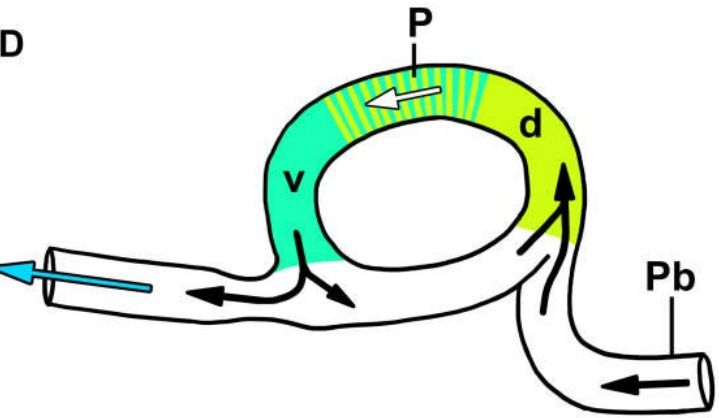




\section{Figure 11}

Figure 11

3D segmented models of the bronchial tree of two live specimens of Alligator mississippiensis (in situ), and three specimens of Crocodylus niloticus generated from $\mu \mathrm{CT}$ and medical grade CT, all in dorsal view. A) The primary, secondary, and tertiary bronchi of a $2.8 \mathrm{~kg}$ A. mississipiensis; B) the primary, secondary, and tertiary bronchi of a $11 \mathrm{~kg}$ A. mississippiensis; C) the primary, secondary, and tertiary bronchi of a $0.5 \mathrm{~kg}$ C. niloticus (NNC9); D) the primary, secondary, and tertiary bronchi of a $0.8 \mathrm{~kg}$ C. niloticus (NNC6); E) the primary, secondary, and tertiary bronchi of a $0.9 \mathrm{~kg}$ C. niloticus (NNC5). Images not to scale. Color scheme: white, trachea and primary bronchi; mint green, cervicoventrobronchi (CVB); lime, D2; neon green, D3; aqua, D4; light aqua, D5; light blue, D6, periwinkle, D7; blue, laterobronchi; purple, caudal group bronchi (CGB); red, M1; neon pink, M2; medium pink, M3; light pink, M4; pale pink, M5; pale purple-deep pink-purples, M6-8; yellow-gold, cardiac lobes. 


\section{A: Alligator}

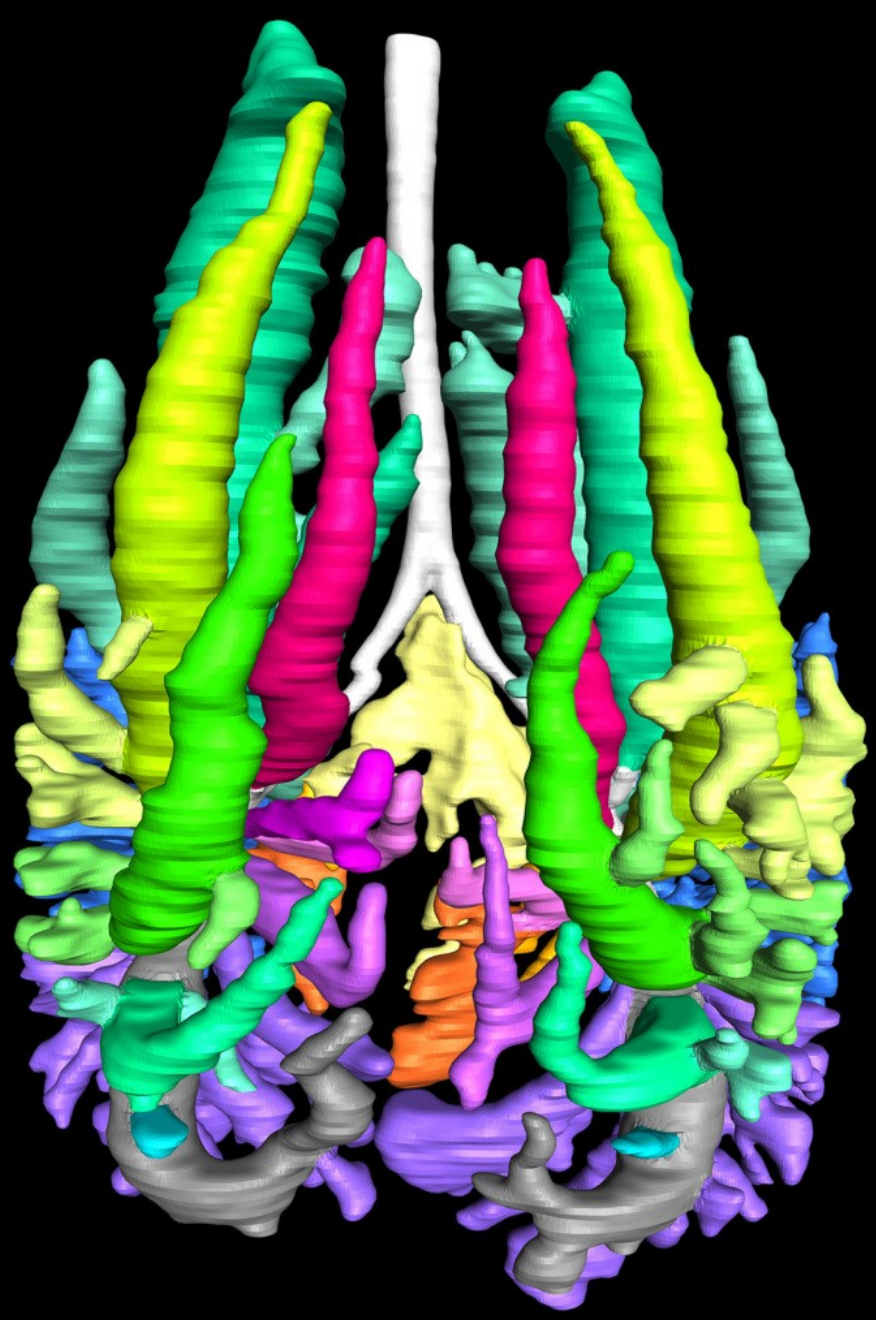

B: Alligator

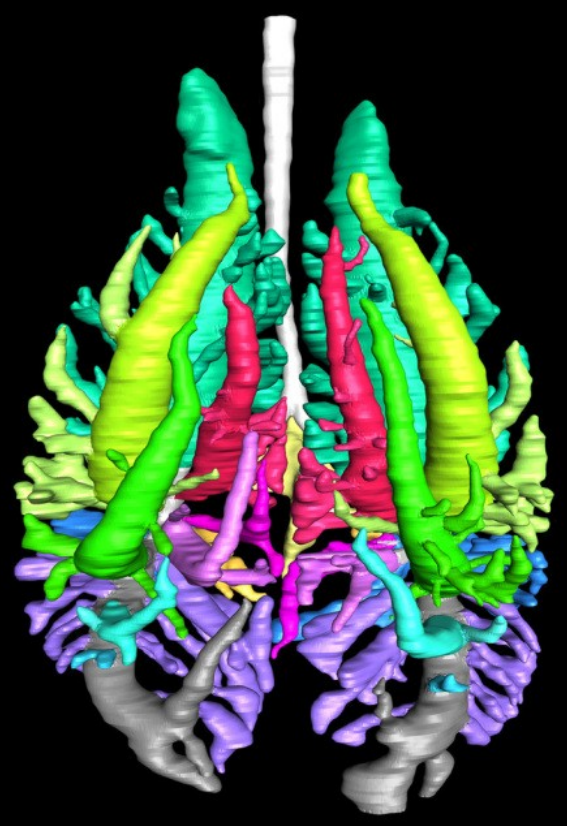

C: Crocodylus

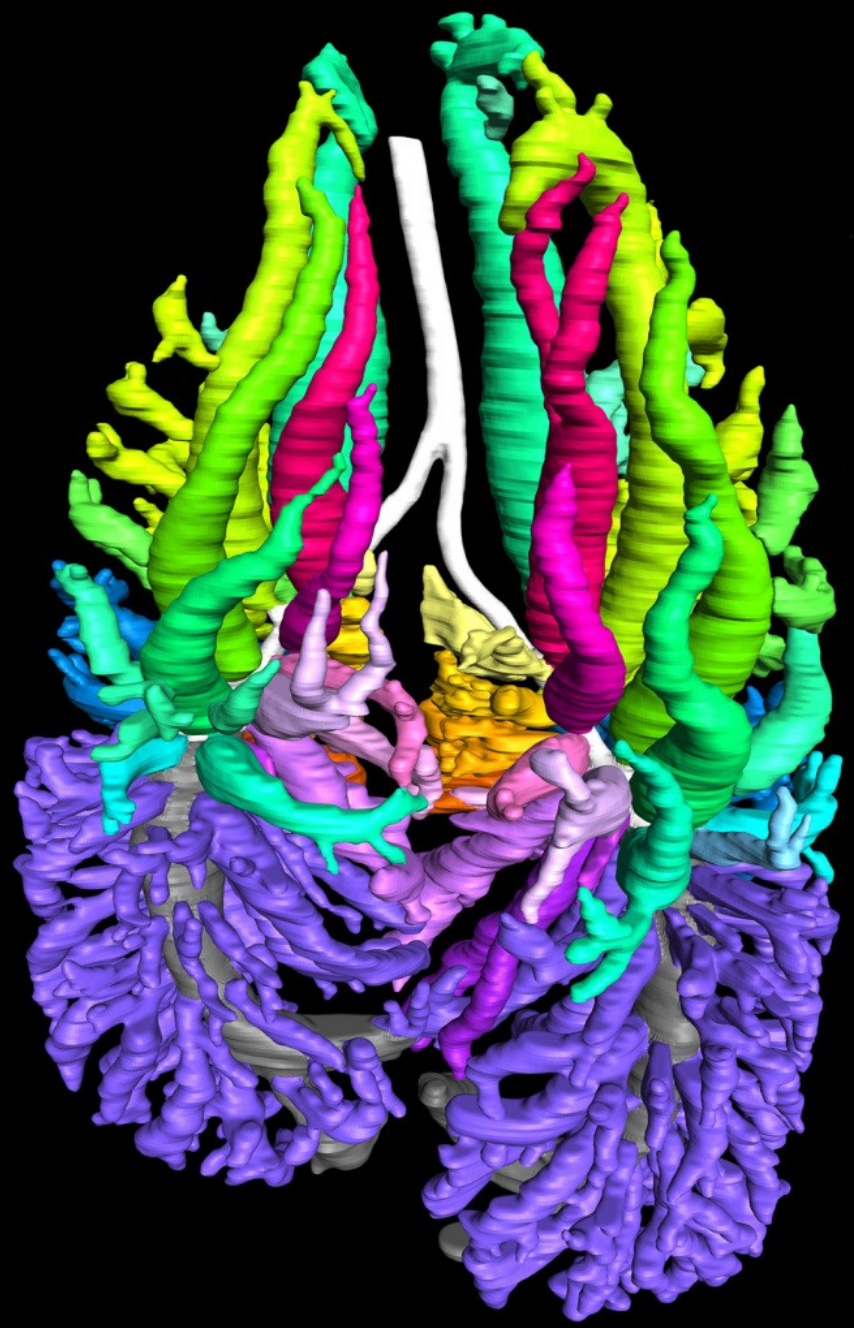

E: Crocodylus
D: Crocodylus

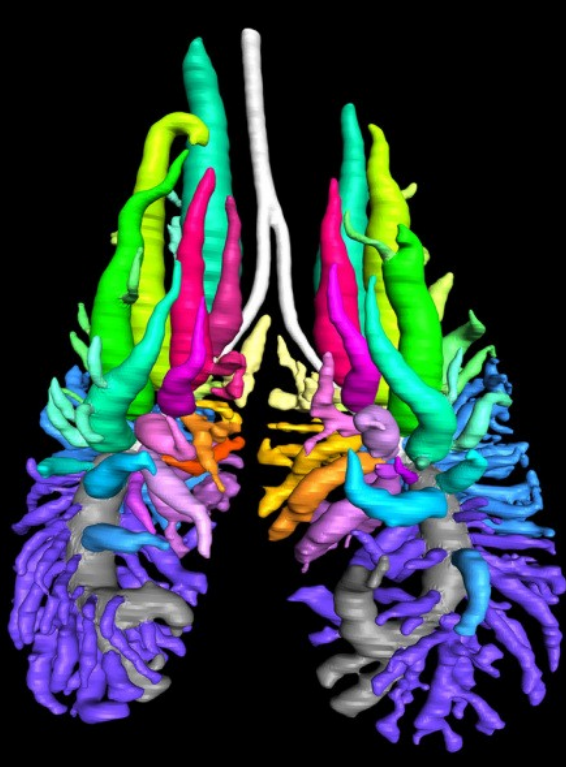

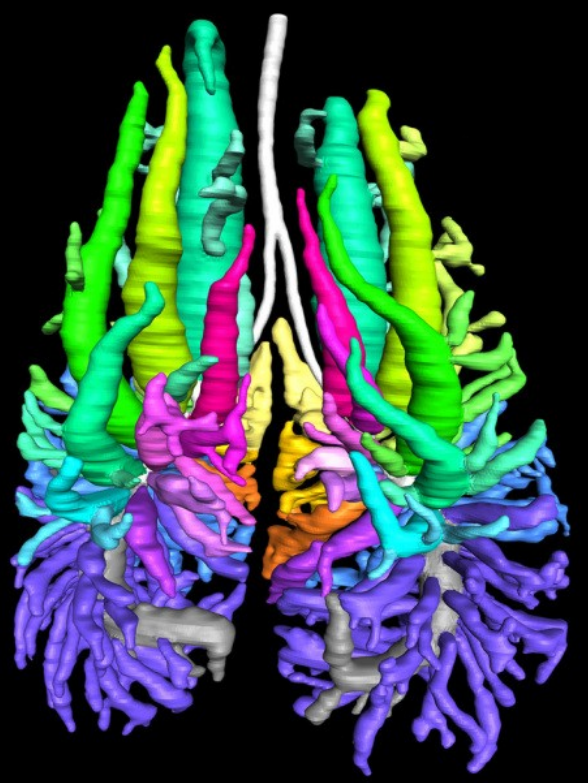




\section{Figure 12}

Figure 12

3D segmented models of the bronchial tree of two live specimens of A. mississippiensis (in situ) and three cadaveric specimens of Crocodylus niloticus generated from $\mu \mathrm{CT}$ and medical grade CT, all in left lateral view. A) The primary, secondary, and tertiary bronchi of a $2.8 \mathrm{~kg}$ A. mississippiensis; B) the primary, secondary, and tertiary bronchi of a $11 \mathrm{~kg}$ A. mississippiensis; C) the primary, secondary, and tertiary bronchi of a $0.5 \mathrm{~kg}$ C. niloticus (NNC9); D) the primary, secondary, and tertiary bronchi of a $0.8 \mathrm{~kg}$ C. niloticus (NNC6); E) the primary, secondary, and tertiary bronchi of a $0.9 \mathrm{~kg}$ C. niloticus (NNC5). Images not to scale. Color scheme: white, trachea and primary bronchi; mint green, cervicoventrobronchi (CVB); lime, D2; neon green, D3; aqua, D4; light aqua, D5; light blue, D6, periwinkle, D7; blue, laterobronchi; purple, caudal group bronchi (CGB); red, M1; neon pink, M2; medium pink, M3; light pink, M4; pale pink, M5; pale purple-deep pink-purples, M6-8; yellow-gold, cardiac lobes. 

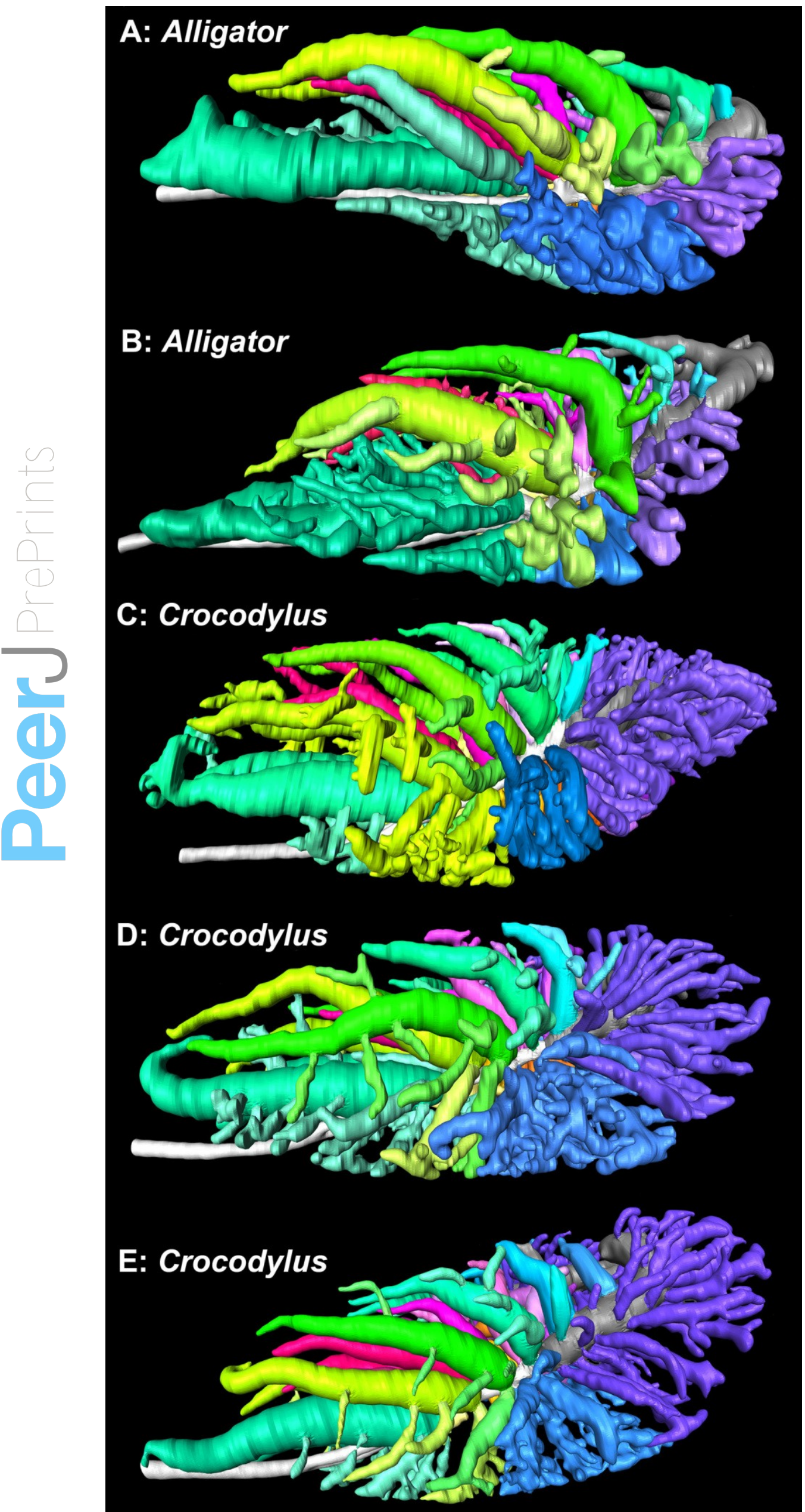


\section{Figure 13}

Figure 13

Diagrammatic representations of the crocodilian (A) and avian (B) lungs in left lateral view with colors identifying proposed homologous characters within the bronchial tree and air sac system of both groups. The image of the bird is modified from Duncker (1971). Abbreviations: AAS, abdominal air sac; CAS, cervical air sac; CRTS, cranial thoracic air sac; CSS, caudal sac-like structure; CTS, caudal thoracic air sac; d, dorsobronchi; GL, gas-exchanging lung; HS, horizontal septum; L, laterobronchi; NGL, non-gas-exchanging lung; ObS, oblique septum; P, parabronchi; $\mathrm{Pb}$, primary bronchus; $\mathrm{Tr}$, trachea; v, ventrobronchi.

A
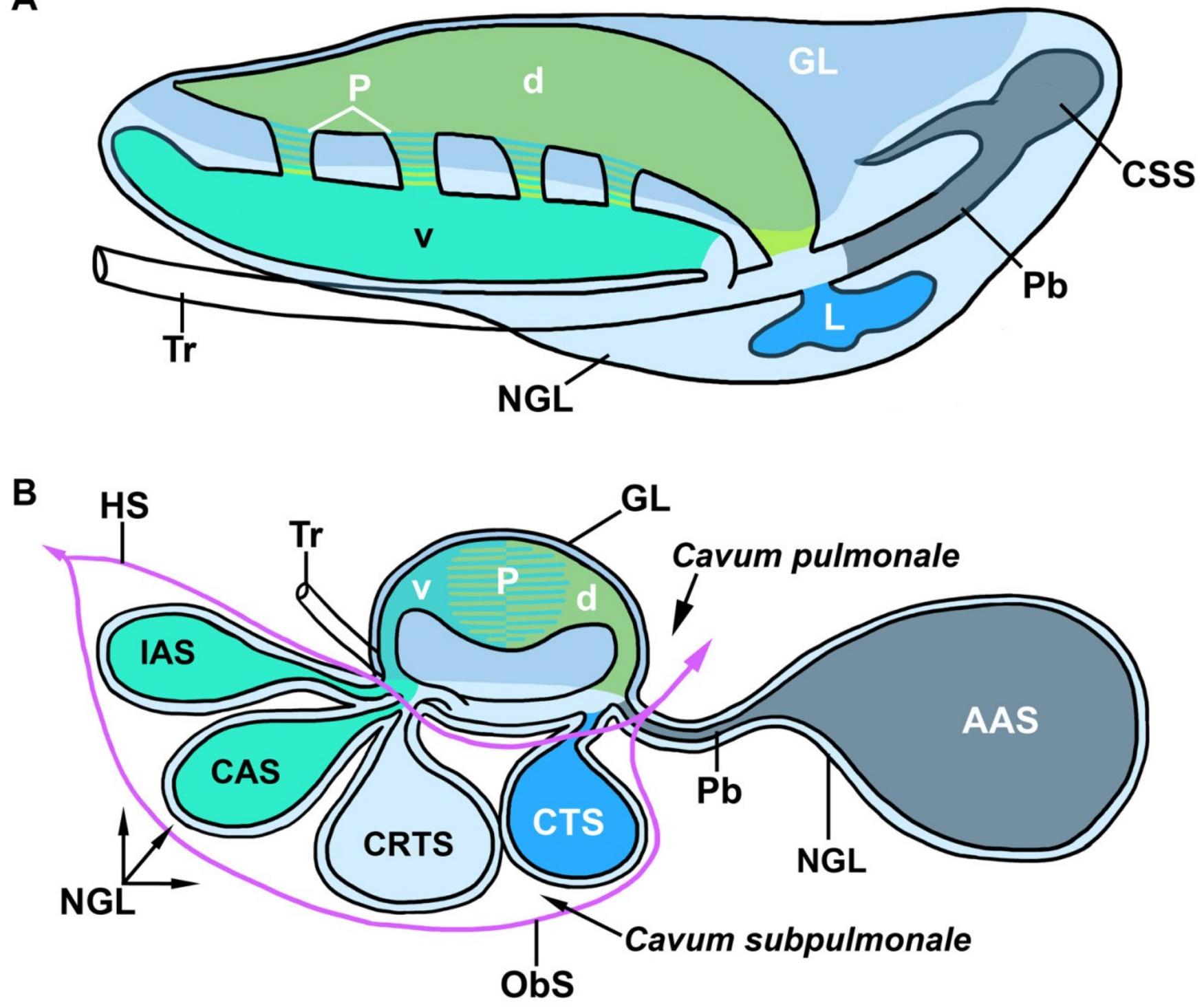